\title{
Correlation Theory of a Two-Dimensional Plasma Turbulence with Shear Flow
}

\author{
Y.Z. Zhang and S.M. Mahajan \\ Institute for Fusion Studies \\ The University of Texas at Austin \\ Austin, Texas 78712 \\ DOE/ET/53088--570 \\ DE93 000880
}

\begin{abstract}
When the ion sound effect is neglected, a wide class of electrostatic plasma turbulence can be modelled by a two-dimensional equation for the generalized enstrophy $\Psi$, an inviscid constant of motion along the turbulent orbits. Under the assumption . of a Gaussian stochastic electrostatic potential, an averaged Green's function method is used to rigorously derive equations for the $N$-particle correlation functions for a dissipative and sheared flow. This approach is equivalunt to the cumulant expansion method [T.H. Dupree, Phys. Fluids 15, 334 (1972); 21, 783 (1978)] used to study the Vlasov-Poisson system. For various cases of interest, appropriate equations are solved to obtain the absolute level as well as the detailed structure of the two-point correlation function $C(\mathbf{r})$, and its Fourier transform, the enstrophy spectral function $I(\mathbf{k})$. Uniformly valid analytical expressions are derived for the dissipative but shearless case resulting in a 'fluctuation-dissipation' theorem relating the total spectral intensity to classical viscosity. These self-consistent results show a strong logarithmic modification of the mixing length estimates for the turbulence levels. For the extremely important and interesting problem of a sheared flow, the suppression of turbulence is demonstrated by using asymptotic analytical technicues in the inviscid range, and uniformly

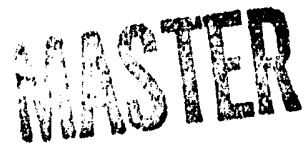


valid numerical methods for the dissipative system. The current asymptotic methods reproduce the results obtained in the orbit picture [Y.Z. Zhang and S.M. Mahajan, Phys. Fluids B 4, 1385 (1992)], but provide much clearer physical perspective and a better definition of crucial parameters like the decorrelation time. The uniformly valid numerical approach allows the determination of the change in spectral shape and intensity due to the presence of shear. It is found that the suppression is more effective for longer wavelengths leading to a spectral shift towards the shorter wavelengths. This and other relevant issues, concerning che role of flows with shear (including its radial variation) in the understanding of the $\mathrm{L}-\mathrm{H}$ transitions in tokamaks, are discussed. 


\section{Introduction}

The theory of turbulence with shear flow has received much attention recently because of its possible relevance to high confinement experiments in tokamaks. A strong correlation has been discovered between a sudden increase in poloidal rotation near the plasma edge and the transition from the low confinement $(\mathrm{L})$ to the high confinement $(\mathrm{H})$ phase. ${ }^{1-5}$ The transition is characterized by a rapid improvement in the particle and energy confinement in the region. The possibility of triggering and sustaining this highly desirable H-phase may be an important siep towards the success of the controlled thermonuclear program in the near future.

There is an overwhelming consensus that the improved confinement is due to the suppression of turbulence resulting in reduced anomalous (turbulence induced) transport. Although the experiments have not yet established a detailed causal connection between the appearance of shear flow and the reduction of turbulence levels, it is strongly felt that such a connection does exist. ${ }^{6-8}$

A variety of theoretical investigations have been made to examine and analyze possible mechanisms responsible for the quelling of turbulence by shear flow. A class of theoretical models based on the linear stability analysis ${ }^{9-11}$ have had some success in showing the existence of stability windows [for particular modes] in parameters associated with poloidal rotations including the shear, and the radial variation of shear. Realizing, however, that the level of observed turbulence is so high in the edge region, it is difficult to believe that the linear theories would provide a deep understanding of this complex phenomenon. There has also been a considerable amount of work involving turbulence models. Most turbulence theories (independent of the details of underlying modes and instabilities) ${ }^{12-16}$ share a very simple physical picture which could lead to the shear induced suppression: For an invariant 
source driving the steady-state turbulence the shear flow reduces the decorrelation time of two turbulent fluid elements resulting in a decrease in the correlation function (which is a measure of the turbulent intensity).

This simple picture, however, may encounter a consistency problem. The assumption of an invariant source strength is applicable only for a time scale much shorter than the transport time scale. It is well known that in the H-phase, the equilibrium density and temperature gradients at the plasma edge become much steeper, implying a possible significant enhancement of the source during the later stage of the H-phase. It is also observed that the effective suppression of shear flow becomes weaker as the equilibrium gradients are increased. ${ }^{16}$ The maintenance of the turbulence suppression is possible in this scenario, only if the turbulence spectrum is shifted towards shorter wavelengths by the shear flow, and/or the suppression is more effective in longer wavelengths, so that the total source, as a combination of the equilibrium gradients and the wavenumber spectrum would not change significantly. ${ }^{16}$ Unfortunately, most previous studies ${ }^{12-16}$ (valid only in the inviscid asymptotic short wave length region) cannot answer these challenging questions. In fact, it would appear that the previous proofs of turbulence suppression may pertain to the uninteresting part of the spectrum; no honest statements could be made for the bulk of the spectrum.

The above-mentioned inadequacies of previous studies ${ }^{12-16}$ demand a more comprehensive development of the correlation theory, a prototype of which was proposed by Dupree for the Vlasov-Poisson system" ${ }^{17,18}$ (The terminology "clump" in Refs. 17 and 18 may suggest validity only in an asymptotic form at small correlation distance). The theory was constructed for the temporal evolution of the correlation function of Vlasov distributions under the influence of turbulent electric fields, as if the latter are "external fields." This does not necessarily imply a violation of self-consistency, if the theoretical problem is treated properly. The main simplicity of the theory arises from the fact that the Vlasov distribution is a constant of motion along the exact orbits, implying a $\delta$-function type of Green's function 
for an initial value problem. This suggests a choice of our basic physical model, to which the correlation theory might be usefully extended.

Before describing the physical model and the contents of this paper, it is worth mentioning that the correlation theory of two dimensional (2D) turbulence developed in this paper has a much greater scientific constituency than the explanation of tokamak edge physics. We hope it will find many useful applications.

We show in Sec. Il, that when the ion sound effect can be neglected in the dynamics described by two-fluid moment equations, the generalized enstrophy (defined as a linear combination of the logarithm of density, and the vorticity) becomes a constant of motion (similar to the Vlasov distribution mentioned earlier) in the inviscid range. The equation for the generalized enstrophy is not necessarily a self-closed one, very much like the Vlasov equation, save for the special case of the Hasegawa-Mima equation. ${ }^{19,20}$ Although the theory would be simpler in the inviscid range, it is necessary to include viscosity (however small) in order to insure the saturation of enstrophy, so that a steady state of turbulence can be achieved. ${ }^{21}$ The inclusion of finite dissipation immediately destroys the ideal property of the enstrophy as a constant of motion. The generalization of the correlation theory to include finite dissipation is, in fact, straightforward, once we realize that the essence of Dupree's theory ${ }^{17,18}$ merely lies in the assumption of a Gaussian stochastic process for the "external fields." For future convenience, a systematic Green's function method, which has already been developed for some time, ${ }^{22}$ is presented, so that the paper is self-contained. This approach is essentially equivalent to the cumulant expansion method adopted by Dupree, ${ }^{17,18}$ and is developed in Sec. III, where we derive a uniformly valid explicit equation governing the evolution of the 2-point correlation function $C(\mathbf{r})$.

Central to this derivation is the expressions for the turbulent diffusion coefficient which happens to be exactly the one given by Dupree. ${ }^{18}$ This result, however, is shown to be quite rigorous on time scales much longer than the decorrelation time. Note that in Dupree's 
derivation, it would appear that some unclarified approximations are made ${ }^{18}$ Comparative rigor in this derivation follows from the divergenceless nature of the $\mathbf{E} \times \mathbf{B}$ motion; it is not surprising (for the same reason) that the usual non-Markovian, or the drag term, appearing in the one-dimensional Vlasov-Poisson system ${ }^{22}$ does not appear in our model $2 \mathrm{D}$ problem. The details are given in Appendix A.

In Sec. IV the equation governing the correlation function is treated in the shearless viscous case to obtain uniformly valid solutions for all correlation distance $r=|\mathbf{r}|$. Appropriate expressions for the spectrum function (Fourier-Bessel transforms of the correlation function) valid in a broad range of $k$ are obtained. An exact (for the homogeneous turbulence) 'fluctuation-dissipation' theorem relating the turbulence level to the viscosity coefficient $(\mu)$ is derived; the appearance of the logarithmic term $[\ln \mu]$ significantly modifies the well-known mixing length scaling. Explicit expressions for the total intensity $\mathcal{E}$ and the diffusion coefficient $D$ are obtained after invoking a self-consistent parametric relation between the two. This folding-in of the spectrum into $D$ clearly brings out the fact that both $\mathcal{E}$ and $D$ depend logarithmically on the viscosity coefficient.

Examination of the effects of shear on the turbulence level as well as on the nature of the turbulence spectrum is one of the major goals of this effort. In Sec. V we obtain asymptotic solutions for the correlation function $C(\mathbf{r})$ for small $r$, and the spectral function $I(\mathbf{k})$ [an exact integral equation for $I(\mathbf{k})$ is also displayed] for large $\mathbf{k}$ for the inviscid system with arbitrary sheas. It is shown that with the rise of shear, the spectrum goes from a near isotropic to a dumbbell-like shape. The behavior of the correlation function with shear is also displayed.

In Chapter VI, we develop a global theory to find answers to the more challenging questions posed earlier. In contrast to the model independent approach in the shearless theory, for this case we have to assume a model for the 'external' spectrum. It turns out the results are quite insensitive to the details of the model as long as some general criteria are met. 
This is because of the fact that the equations determining $C(\mathbf{r})$ depend on the normalized integrals of the 'external' spectrum, and these integrals for appropriately normalized functions (with acceptable behavior for large $k$ ) do not differ much from one another. As an example, we have worked out the details when a Gaussian spectrum for the 'external' field is assumed. A global calculation for weak to moderate shear is numerically carried out, and displayed in a set of graphs. We find that (in this global calculation), the velocity shear not only suppresses the overall turbulence levels, but also preferentially suppresses the long wavelength part of the spectrum. Naturally both of these are very important results if the shear effects are to account for the phenomonology of the L-H transition.

In Sec. VII we compare the present work with 'orbit' theory developed earlier ${ }^{12-16}$ and compare and contrast the two approaches. In fact, it is only through the current approach, that a meaningful quantitative definition of the decorrelation time (essential to the orbit theory) can be given.

Section VIII summarizes the main results of this paper and also points out the basic assumptions made in this effort. Algebraic details of some parts of the paper are worked out in Appendices A-C.

\section{Physical Model for a 2-D Plasma Turbulence}

When ion parallel dynamics can be neglected, the low frequency electrostatic motion of a magnetized plasma is effectively limited to the 2D surface perpendicular to the magnetic field. The basic system is then composed of the electron continuity equation and the equation for quasineutrality. The electron continuity equation for the number density $(n)$ is

$$
\frac{\partial}{\partial t} n+\nabla \cdot\left(n \mathbf{v}_{e}\right)=0
$$

where the electron velocity $\mathbf{v}_{e}=v_{e, \|} \mathbf{b}+(c / B) \mathbf{b} \times \nabla \Phi-(c / B e n) \mathbf{b} \times \nabla P_{e}+$ ("classical" diffusion term), $\Phi$ is the total electrostatic potential, consisting of the equilibrium $\phi_{0}$ and 
the fluctuating $\phi, \mathbf{b}$ is the unit vector along the magnetic field (with magnitude $B$ ), $c$ is the speed of light, $P_{e}$ is the electron pressure, $e>0$ is the elementary charge, and the "classical" diffusion term is an artifact added for constructing the final model equation [Eq. (4)].

The equation for quasineutrality or charge conservation $(\boldsymbol{\nabla} \cdot \mathbf{J}=0)$ is conveniently written as

$$
\nabla_{\|} j_{\|}+\nabla_{\perp} \cdot \mathbf{j}_{\perp}=0
$$

with the divergence of the perpendicular current [in the cold ion limit] given by

$$
\nabla_{\perp} \cdot \mathbf{j}_{\perp}=-m_{i} n\left(\frac{c}{B}\right)^{2} \frac{d}{d t} \nabla_{\perp}^{2} \Phi+\nu \nabla_{\perp}^{4} \Phi
$$

where $\nu$ is the perpendicular viscosity coefficient, $m_{i}$ is the ion mass, the comoving derivative $d / d t \equiv \partial / \partial t+(c / B) \mathbf{b} \times \nabla \Phi \cdot \nabla$. When ion parallel motion is neglected, the parallel current $j_{\|} \simeq-e n v_{e, \|}$. With this definition, Eqs. (1)-(3) can be manipulated to eliminate $j_{\|}$, and yield the single nonlinear equation for the hybrid field $\Psi$,

$$
\left[\frac{\partial}{\partial t}+\frac{c}{B}(\mathbf{b} \times \nabla \Phi) \cdot \nabla-\mu \nabla^{2}\right] \Psi \equiv\left(\frac{d}{d t}-\mu \nabla^{2}\right) \Psi(\mathbf{r}, t)=0 .
$$

The field $\Psi \equiv \ln n-\nabla_{\perp}^{2} \Phi$ is called the generalized enstrophy. In obtaining Eq. (4) an appropriate "classical" diffusion term has been chosen in combination with the perpendicular viscosity for constructing the dissipative term proportional to $\mu$. In the rest of this paper, all lengths are normalized to $\rho_{s} \equiv c_{s} / \omega_{c i}$, and the time scale is normalized to $1 / \omega_{c i}$, where $c_{s} \equiv$ $\sqrt{T_{e} / m_{i}}$ is the ion sound speed, $T_{e}$ is the electron temperature, and $\omega_{c i}$ is the ion cyclotron frequency. The potential $\Phi$ is also normalized to the electron thermal energy. Equation (4) can also be derived from the ion continuity equations with the same assumptions.

The most remarkable feature of $\mathrm{Eq} .(4)$ is that it is independent of any particular form for the parallel Ohm's law. It can be viewed as one of the Hasegawa-Wakatani equation set ${ }^{21}$ describing the collisional drift wave (with an artificial "classical" diffusion) as well as the Hasegawa-Mima equation, ${ }^{19,20}$ describing the collisional drift wave, if an adiabatic electron 
density response is assumed. Since we have not assumed constancy of electron temperature in the derivation, Eq. (4) may also describe the dissipative drift wave $e^{23-25}$ for which the ion sound is not important. When the dissipative term in Eq. (4) is ignored, the generalized enstrophy becomes a constant of motion along the perturbed orbit. Another important feature of the model is that the dynamics associated with the magnetic shear does not enter into the basic description [Eq. (4)] of turbulence. It seems that neglecting the ion sound effect obviates the need for a detailed Ohm's law, and further makes the system independent of magnetic shear. This results in a crucial simplification of the entire problem.

There exist other plasma phenomena whose mathematical formulation leads to equations similar to Eq. (4). Such models, for example, have been constructed for the rippling-like modes. ${ }^{26}$ For these modes the parallel dissipation given by $\chi_{\|} \nabla_{\|}^{2} \Psi$ (where $\chi_{\|}$is the parallel heat conduction) is dominant and replaces the perpendicular dissipation term $\mu \nabla^{2} \Psi$ in Eq. (4). The field $\Psi$ may represent either the electron temperature, or the effective impurity charge $Z_{\text {eff }}$. The parallel dissipation, however, unlike the viscous dissipation (which is important only at short distances) renders the whole spectral range to be dissipative. Naturally, the methodology to deal with this type of dissipation is somewhat different from that depicted in the present paper, and will be presented elsewhere.

Throughout this paper, it is assumed that the equilibrium density and the electrostatic potential vary only in the radial direction. As a result, the radially sheared flow is in the direction perpendicular both to the radial, and to the direction of the magnetic field.

\section{The Averaged Green's Function Method in a Gaussian Stochastic Field $\phi$}

In this section we shall derive the equation of evolution of a general equal time spatial correlation function. The $N$ 'body' correlation function, denoted by $\left\langle\Psi\left(\mathbf{r}_{1}, t\right) \Psi\left(\mathbf{r}_{2}, t\right) \ldots \Psi\left(\mathbf{r}_{N}, t\right)\right\rangle$, where $(\ldots\rangle)$ is the ensemble average on realizations of turbulence, will be constructed by 
letting $\Psi$ evolve under the influence of a Caussian stochastic field $\phi$, and treating $\phi$ as an external field. Notice that while the "external field" $\phi$ is assumed to be Gaussian, the response (the fluctuating part of $\Psi$ ) is not necessarily so.

The exact time evolution of generalized enstrophy satisfying Eq. (4) with the initial condition $\Psi\left(\mathbf{r}, t=t_{0}\right)=\Psi_{0}(\mathbf{r})$, can be expressed in terms of a Green's function:

$$
\Psi(\mathbf{r}, t)=\int d \mathbf{r}^{\prime}\left(\boldsymbol{r}\left(\mathbf{r}, \mathbf{r}^{\prime} ; t, t_{0}\right) \Psi_{0}\left(\mathbf{r}^{\prime}\right)\right.
$$

where

$$
\begin{aligned}
G\left(\mathbf{r}, \mathbf{r}^{\prime} ; t, t_{0}\right) & \equiv \frac{\pi}{\mu\left(t-t_{0}\right)} \exp \left(-\frac{\left[\mathbf{r}-\mathbf{r}^{\prime}-\int_{t_{0}}^{t} d s \mathbf{b} \times \nabla \Phi(r(s), s)\right]^{2}}{4 \mu\left(t-t_{0}\right)}\right) \\
& =\int d \mathbf{k} \exp \left(-\mu k^{2}\left(t-t_{0}\right)+i \mathbf{k} \cdot\left[\mathbf{r}-\mathbf{r}^{\prime}-\int_{t_{0}}^{t} d s \mathbf{b} \times \nabla \Phi(r(s), s)\right]\right),
\end{aligned}
$$

with the full orbit given by $\mathbf{r}(t) \equiv \mathbf{r}\left(t_{0}\right)+\int_{t_{0}}^{t} d s \mathbf{h} \times \nabla \Phi(\mathbf{r}, t)=\mathbf{r}$. By direct substitution, the interested reader can verify that $\mathrm{Eq}_{\mathrm{q}} \mathrm{s}$. (5)-(6), indeed, formally solve Eq. (4). Notice that Eq. (5) expresses $\Psi(\mathbf{r}, t)$ in terms of the 'external field' $\Phi$.

Let us first consider the $N=1$ case, i.e., calculate the time evolution of the ensemble average of $\Psi,\langle\Psi(\mathbf{r}, t)\rangle$. Ensemble averaging over Eq. (4) yields

$$
\left(\frac{\partial}{\partial t}+\mathbf{b} \times \nabla \phi_{0}(\mathbf{r}, t) \cdot \nabla-\mu \nabla^{2}\right)\langle\Psi(\mathbf{r}, t)\rangle+\langle\mathbf{b} \times \nabla \phi(\mathbf{r}, t) \cdot \nabla \Psi(\mathbf{r}, t)\rangle=0 .
$$

Since $\Psi(\mathbf{r}, t)$ is known as a functional of $\phi$, it is straightforward to evaluate the second term of Eq. (7) by invoking the Gaussian assumption for the stochastic field $\phi$, and using the well-known formula, ${ }^{27-28}$

$$
\langle\phi(x) F[\phi]\rangle=\int d x^{\prime}\left\langle\phi(x) \phi\left(x^{\prime}\right)\right\rangle\left\langle\frac{\delta}{\delta \phi\left(x^{\prime}\right)} F[\phi]\right\rangle,
$$

where $F[\phi]$ is a functional of $\phi, x$ stands for all the arguments of the function $\phi$, including the indices over which it is necessary to sum. Equation (4) can be proved, for example, in the special case when $F[\phi]$ can be expanded in a functional Taylor series. This procedure represents 
a generalization of the well-known formula for the correlation of a Gaussian random quantity $\chi$ (could be a continuous field) with a function of this quantity $f(\chi):\langle\chi f(\chi)\rangle=\left\langle\chi^{2}\right\rangle\left\langle f^{\prime}(\chi)\right\rangle$ $[$ for $\langle x\rangle=0]$.

In order to use formula (8) to calculate the last term in $\mathrm{Eq}$. (7), we need the functional derivative of $\Psi(\phi)$,

$$
\begin{aligned}
\frac{\delta \Psi\left(\mathbf{r}_{i}, t\right)}{\delta \phi\left(\mathbf{r}^{\prime}, t^{\prime}\right)} & =\int d \mathbf{r}^{\prime \prime} d \mathbf{k} \exp \left(-\mu k^{2}\left(t-t_{0}\right)+i \mathbf{k} \cdot\left[\mathbf{r}_{i}-\mathbf{r}^{\prime \prime}-\int_{t_{0}}^{t} d s \mathbf{b} \times \nabla_{i} \Phi\left(\mathbf{r}_{i}(s), s\right)\right]\right) \\
& \cdot(-i \mathbf{k}) \cdot \int_{t_{0}}^{t} d s \mathbf{b} \times \nabla_{i} \delta\left[\mathbf{r}^{\prime}-\mathbf{r}_{i}(s)\right] \delta\left(t^{\prime}-s\right) \Psi_{0}\left(\mathbf{r}^{\prime \prime}\right) \\
& =-\theta\left(t-t^{\prime}\right) \mathbf{b} \times \nabla_{i} \delta\left[\mathbf{r}^{\prime}-\mathbf{r}_{i}\left(t^{\prime}\right)\right] \cdot \nabla_{i} \Psi\left(\mathbf{r}_{i}, t\right),
\end{aligned}
$$

where $\boldsymbol{\nabla}_{i}=\partial / \partial \mathbf{r}_{i}$, and $\theta\left(t-t^{\prime}\right)$ is the Heaviside step function reflecting a causal response of $\Psi$ to $\phi$. The use of Eqs. (8)-(9) converts the term $\langle b \times \nabla \phi(\mathbf{r}, t) \cdot \nabla \Psi(\mathbf{r}, t)\rangle$ of Eq. (7) into a diffusive term $\equiv-\nabla_{i} \cdot \mathbf{D}_{i i} \cdot \nabla_{i}\left\langle\Psi\left(\mathbf{r}_{i}, t\right)\right\rangle$, where

$$
\mathbf{D}_{i j} \equiv \int_{t_{0}}^{t} d t^{\prime}\left\langle\mathbf{b} \times \nabla_{i} \phi\left(\mathbf{r}_{i}(t), t\right) \mathbf{b} \times \nabla_{j} \phi\left(\mathbf{r}_{j}\left(t^{\prime}\right), t^{\prime}\right)\right\rangle,
$$

is the turbulent diffusion tensor generated by the correlations of the fluctuating fields (for $i, j=1 \ldots N)$. Here, and henceforth, we use the Latin letters to denote spatial positions, and Greek letters to denote components of the Cartesian coordinates. Thus, Eq. (7) for $\left\langle\Psi\left(\mathbf{r}_{i}, t\right)\right\rangle$ takes the form

$$
\left(\frac{\partial}{\partial t}+\mathbf{b} \times \nabla_{i} \phi_{0}\left(\mathbf{r}_{i}, t\right) \cdot \nabla_{i}-\mu \nabla_{i}^{2}-\nabla_{i} \cdot \mathbf{D}_{i i} \cdot \nabla_{i}\right)\left\langle\Psi\left(\mathbf{r}_{i}, t\right)\right\rangle=0, \quad .
$$

and describes the evolution of ensemble average of the total enstrophy, in particular, under the influence of turbulence-induced diffusion. Naturally, $\mathbf{D}_{\mathfrak{t}}$ are generally interpreted to be the turbulence diffusion coefficients. Equation (11) can allow a steady state for a constant radial gradient of $\langle\Psi(\mathbf{r}, \infty)\rangle$, if uniforınity in the poloidal direction is assumed.

The time evolution for the two-point correlation function $\left\langle\Psi\left(\mathbf{r}_{1}, t\right) \Psi\left(\mathbf{r}_{2}, t\right)\right\rangle(N=2$ case $)$ 
is derived in a similar manner. Starting with

$$
\frac{\partial}{\partial t}\left\langle\Psi\left(\mathbf{r}_{1}, t\right) \Psi\left(\mathbf{r}_{2}, t\right)\right\rangle=\left\langle\frac{\partial \Psi\left(\mathbf{r}_{1}, t\right)}{\partial t} \Psi\left(\mathbf{r}_{2}, t\right)\right\rangle+\left\langle\Psi\left(\mathbf{r}_{1}, t\right) \frac{\partial \Psi\left(\mathbf{r}_{2}, t\right)}{\partial t}\right\rangle,
$$

and substituting Eq. (4) into (12), we find that the term

$$
Q_{i} \equiv\left\langle\mathbf{b} \times \nabla_{i} \phi\left(\mathbf{r}_{i}, t\right) \cdot \nabla_{i} \Psi\left(\mathbf{r}_{1}, t\right) \Psi\left(\mathbf{r}_{2}, t\right)\right\rangle \quad(i=1,2)
$$

needs to be calculated. A straightforward application of Eqs. (6)-(9) yields

$$
Q_{i}=\left(\nabla_{i} \cdot \mathbf{D}_{i 1} \cdot \nabla_{1}+\nabla_{i} \cdot \mathbf{D}_{i 2} \cdot \nabla_{2}\right)\left\langle\Psi\left(\mathbf{r}_{1}, t\right) \Psi\left(\mathbf{r}_{2}, t\right)\right\rangle \quad(i=1,2)
$$

which leads to the following equation for $\left\langle\Psi\left(\mathbf{r}_{1}, t\right) \Psi\left(\mathbf{r}_{2}, t\right)\right\rangle$,

$$
\left(\frac{\partial}{\partial t}+\sum_{i=1,2}\left(\mathbf{b} \times \nabla_{i} \phi_{0}\left(\mathbf{r}_{i}, t\right) \cdot \nabla_{i}-\mu \nabla_{i}^{2}\right)-\sum_{i, j=1,2} \nabla_{i} \cdot \mathbf{D}_{i j} \cdot \nabla_{j}\right)\left\langle\Psi\left(\mathbf{r}_{1}, t\right) \Psi\left(\mathbf{r}_{2}, t\right)\right\rangle=0,
$$

where $\mathbf{D}_{i j}$ 's have already been defined by Eq. (10). In Appendix A, we calculate an expression for $\mathbf{D}_{i j}$ in terms of the spectrum of the "external" field $\phi$ for a stationary and homogeneous turbulent system, and show that the commonly used expression ${ }^{18}[\mathrm{Eq} .(\mathrm{A} 17)]$ is a rigorous result under the assumption of homogeneity.

The above derivation for $N=1,2$ can be readily generalized to derive appropriate equations for higher correlations; the $N$ particle correlation obeys

$$
\left\langle\frac{\partial}{\partial t}+\sum_{i=1}^{N}\left(\mathbf{b} \times \nabla_{i} \phi_{0}\left(\mathbf{r}_{i}, t\right) \cdot \nabla_{i}-\mu \nabla_{i}^{2}\right)-\sum_{i, j=1}^{N} \nabla_{i} \cdot \mathbf{D}_{i j} \cdot \nabla_{j}\right)\left\langle\prod_{i=1}^{N} \Psi\left(\mathbf{r}_{i}, t\right)\right\rangle=0 .
$$

In the inviscid limit $(\mu \rightarrow 0)$, the Green's function $[\mathrm{Eq} .(6)]$ is just the $\delta$-function

$$
G\left(\mathbf{r}, \mathbf{r}\left(t_{0}\right) ; t, t_{0}\right)=\delta\left[\mathbf{r}-\mathbf{r}\left(t_{0}\right)-\int_{t_{0}}^{t} d s \mathbf{b} \times \nabla \Phi(\mathbf{r}(s), s)\right]
$$

and the enstrophy becomes a constant of motion along the perturbed orbits. The $N$-point correlation function is then simply the average of a product of $N$ delta functions [the relevant Green's functions],

$$
\Gamma_{N} \equiv\left\langle\prod_{i=1}^{N} \delta\left(\mathbf{r}_{i}-\mathbf{r}_{i}(t)\right)\right\rangle=\left\langle\prod_{i=1}^{N} \delta\left(\mathbf{r}_{i}-\mathbf{r}_{i}\left(t_{0}\right)-\int_{t_{0}}^{t} d s \mathbf{b} \times \nabla_{i} \Phi\left(\mathbf{r}_{i}(s) s\right)\right)\right\rangle
$$


By direct manipulation, it can be readily demonstrated that $\Gamma_{N}$, indeed, satisfies the $\mu=0$ version of Eq. (16) obeyed by $\left\langle\prod_{i=1}^{N} \Psi\left(\mathbf{r}_{i}, t\right)\right\rangle$, i.e.,

$$
\left(\frac{\partial}{\partial t}+\sum_{i=1}^{N} \mathbf{b} \times \nabla_{i} \phi_{0}\left(\mathbf{r}_{i}, t\right) \cdot \nabla_{i}-\sum_{i, j=1}^{N} \nabla_{i} \cdot \mathbf{D}_{i j} \cdot \nabla_{j}\right) \Gamma_{N}=0 .
$$

Once the Green's functions for this system are known, it is straightforward to obtain the averaged values of any relevant observables. One could, in principle, extract useful information about the system even without being able to solve (16) or (19) explicitly. We can, for example, develop the orbit theory, using our implicit $\Gamma_{N}$ 's. The $N$ orbit correlation is defined to be

$$
\left\langle\mathbf{r}_{1}(t) \mathbf{r}_{2}(t) \ldots \mathbf{r}_{N}(t)\right\rangle=\int \prod_{i=1}^{N} d \mathbf{r}_{i}\left(\mathbf{r}_{1} \mathbf{r}_{2} \ldots \mathbf{r}_{N}\right)\left\langle\prod_{i=1}^{N} \delta\left(\mathbf{r}_{i}-\mathbf{r}_{i}(t)\right)\right\rangle
$$

and can be manipulated furth $x$ by using the $\delta$ functions to do the integrals. For the important case of $N=2$, it is convenient to introduce the relative coordinate $\mathbf{r}=\mathbf{r}_{1}-\mathbf{r}_{2} \cdot{ }^{17-18}$ The relative orbit correlation is written as

$$
\left\langle r^{\mu}(t) r^{\nu}(t)\right\rangle=\int d \mathbf{r}_{1} d \mathbf{r}_{2} r^{\mu} r^{\nu}{ }_{i}^{\prime}\left(\mathcal{u}^{\prime}\left(\mathbf{i}_{:}-\mathbf{r}(t)\right) \delta\left(\mathbf{r}_{2}-\mathbf{r}_{2}(t)\right)\right\rangle
$$

where $r^{\mu}(t) \equiv r_{1}^{\mu}(t)-r_{2}^{\mu}(t)$. The evolution equation for the relative orbit correlation is obtained from Eq. (19) $[N=2]$ satisfied by $\Gamma_{2}$,

$$
\begin{aligned}
\frac{\partial}{\partial t}\left\langle r^{\mu}(t) r^{\nu}(t)\right\rangle & =\left\langle r^{\mu}(t) r^{\sigma}(t)\right\rangle\left(\frac{d v^{\nu}}{d r^{\sigma}}\right)_{\mathbf{r}_{+}}+\left\langle r^{\sigma}(t) r^{\nu}(t)\right\rangle\left(\frac{d v^{\mu}}{d r^{\sigma}}\right)_{\mathbf{r}_{+}} \\
& +\int d \mathbf{r}_{1} d \mathbf{r}_{2}\left(D_{-}^{\mu \nu}+D_{-}^{\nu \mu}\right)\left\langle\delta\left(\mathbf{r}_{1}-\mathbf{r}_{1}(t)\right) \delta\left(\mathbf{r}_{2}-\mathbf{r}_{2}(t)\right)\right\rangle
\end{aligned}
$$

where $\mathbf{v} \equiv \mathbf{b} \times \nabla \phi_{0}, \mathbf{D}_{-} \equiv \mathbf{D}_{11}+\mathbf{D}_{22}-\mathbf{D}_{12}-\mathbf{D}_{21}$, and $2 \mathbf{r}_{+} \equiv \mathbf{r}_{1}+\mathbf{r}_{2}$. On using Eq. (A17) of Appendix A, the Cartesian components of the effective relative diffusion coefficient $\mathbf{D}_{\text {- }}$ are found to be

$$
D_{-}^{\mu \nu}=2 \int d \mathbf{k}(\mathbf{b} \times \mathbf{k})^{\mu}(\mathbf{b} \times \mathbf{k})^{\nu} \Pi(\mathbf{k})(1-\cos (\mathbf{k} \cdot \mathbf{r}))
$$


Notice that in the limit $r \rightarrow 0$, Eq. (23) is considerably simplified, i.e., the factor 1 $\cos \mathbf{k} \cdot \mathbf{r} \sim(\mathbf{k} \cdot \mathbf{r})^{2} / 2$. The remarkable consequence is that the term proportional to $D$ 's in Eq. (22) reduces to a term proportional to the moment $\left\langle r^{\rho}(t) r^{\sigma}(t)\right\rangle$, and consequently the set represented by Eq. (18) becomes a closed set for the (second order) moments.

We now go back to Eq. (15) for the two-point correlation function, and cast it fully in terms of the relative $\mathbf{r}=\mathbf{r}_{1}-\mathbf{r}_{2}$, and the center of mass $\mathbf{r}_{+}=\left(\mathbf{r}_{1}+\mathbf{r}_{2}\right) / 2$ coordinates. We also divide the total enstrophy into its equilibrium and fluctuating parts, $\Psi=\Psi_{0}+\psi$ with $\langle\psi\rangle=0, \Psi_{0}=\langle\Psi\rangle$. Transformation from $\mathbf{r}_{1}, \mathbf{r}_{2}$ to $\mathbf{r}, \mathbf{r}_{+}$is quite simple except for the term containing the velocity shear. Since the velocity shear is normally limited to a small edge layer (in the problems of current interest), one can readily affect the transformation by placing the Cartesian axis $\hat{e}_{x}$ and $\hat{e}_{y}\left[\mathbf{r}=\hat{e}_{x} x+\hat{e}_{y} y\right]$ on the local $\hat{e}_{r_{+}}$and $\hat{e}_{\theta_{+}}$axes which are approximately the radial and the poloidal directions of the system. Defining $\mathbf{v}=\mathbf{b} \times \nabla \phi_{0}$, and carrying out the algebra, one obtains $\left[C(\mathbf{r}, t) \equiv\left\langle\psi\left(\mathbf{r}_{1}, t\right) \psi\left(\mathbf{r}_{2}, t\right)\right\rangle\right]$

$$
\left[\frac{\partial}{\partial t}+\left(v_{y}^{\prime}\right) x \frac{\partial}{\partial y}-2 \mu \nabla^{2}-\nabla \cdot \mathbf{D}_{-} \cdot \nabla\right] C(\mathbf{r}, t)=\frac{1}{L_{0}^{2}}\left(D_{12}^{x x}+D_{21}^{x x}\right)
$$

where $\nabla=\partial / \partial \mathbf{r}, v_{y}^{\prime}=\partial v_{y} / \partial r_{+}$represents the local poloidal velocity shear, $L_{0}^{-1}=\left|\partial \Psi_{0} / \partial x\right|=$ $\left.\left|d \ln n_{0} / d x-d\left(d^{2} \phi_{0} / d x^{2}\right) / d x\right|=\mid d \ln n_{0} / d x+d^{2} E_{0} / d x^{2}\right\}$ is a measure of the local equilibrium gradients, $E_{0}$ is the equilibrium electric field, and $D_{12}^{x x}$ is determined from the definition Eq. (A17). The quantities $v_{y}^{\prime}, \mu$ and $L_{0}^{-2}$ are assumed to be independent of $x$ and $y$, and will be treated as such in later considerations.

In order to make further progress, we need to evaluate the coefficients of the turbulent diffusion operator a bit more explicitly. Before we proceed, a few explanatory remarks of a general nature are very much in order.

As stated in the introduction, the correlation theory explored in this paper essentially deals with the solutions of enstrophy under a given 'external field' $\Phi$. In the presence of shear, the enstrophy spectrum (in the rest of this paper, unless stated otherwise, spectrum implies 
enstrophy spectrum) is manifestly anisotropic even for short wavelengths when the shear is sufficiently strong. Since the equilibrium $\left(\Psi_{0}\right)$ is anisotropic, i.e., it has gradients only in the $x\left(\hat{e}_{r_{+}}\right)$direction, one expects that the long wavelength (wavelengths comparable to the gradicit scale $L_{0}$ ) part of the spectrum remains anisotropic even when the shear is absent. We do expect, however, that the short wavelength part of the spectrum will tend to be isotropic in a shearless flow. Therefore, for the theory to span all of the aforementioned possibilities, we introduce ellipticity in the spectrum of $\phi$. The 'effective' spectral function $\Pi(k)=$ $\Pi\left(k_{x}^{2}+\alpha^{2} k_{y}^{2}\right)$, where $\alpha$ describes, albeit qualitatively, the elongation of the $\phi$ spectrum. An appropriately deformed $y$ coordinate is then introduced, and the pair $\bar{k}_{y}=\alpha k_{y}, \bar{y}=y / \alpha$ define a new deformed coordinate system $\left[\overline{\mathbf{r}}=(x, \bar{y})\right.$ with the conjugate $\left.\overline{\mathbf{k}}=\left(k_{x}, \bar{k}_{y}\right)\right]$ in which the spectrum is isotropic. This very deformed coordinate system will be used in all later calculations, and the ellipticity parameter will be absorbed in the coefficients $\left(v_{y}^{\prime}, \mu \ldots\right.$ etc.). For notational simplicity, we continue using $\left(x, y, k_{x}, k_{y}, r\right)$ for $\left(x, \bar{y}, k_{x}, \bar{k}_{y}, \bar{r}\right)$, and do not explicitly display $\alpha$. The effects of $\alpha$ can be recalled at will.

With these clarifying remarks on the notation, the components of $\mathbf{D}_{-}\left(\right.$and $\left.D_{12}^{x x}+D_{21}^{x x}\right)$ can be conveniently defined by $x \equiv r \cos \varphi$, and $y \equiv r \sin \varphi$. In Appendix B various components of the diffusion tensor are explicitly displayed. Once the components of the diffusion tensor are known, one can rewrite $\mathrm{Eq} .(24)$ in polar coordinates $[C \equiv C(r, \varphi, \tau)]$

$$
\begin{aligned}
\left\{-\frac{\partial}{\partial \tau}+\right. & \left.\left.\frac{1}{r} \frac{\partial}{\partial r}\left[1-S_{0}(r)-S_{2}(r)+\eta^{2}\right] r \frac{\partial}{\partial r}+\left[1-S_{0}(r)+S_{2}(r)+\eta^{2}\right)\right] \frac{1}{r^{2}} \frac{\partial^{2}}{\partial \varphi^{2}}\right\} C \\
& -\hat{\beta}_{s}\left(\frac{r}{2} \sin 2 \varphi \frac{\partial}{\partial r}+\cos ^{2} \varphi \frac{\partial}{\partial \varphi}\right) C=-\frac{1}{L_{0}^{2}}\left[S_{0}(r)+S_{2}(r) \cos 2 \varphi\right]
\end{aligned}
$$

where the first set of terms in \{\} correspond to the viscous $\left(\eta^{2}\right)$ and the turbulent diffusion, the second set of terms $\left(\widehat{\beta}_{s}\right)$ are due to the equilibrium velocity shear, and the terms on the right-hand side represent the gradient source. This comparatively simple form of Eq. (25) is mainly due to the fact that $\nabla \cdot D_{-}=0$ in our $2 D$ system. Various quantities occurring in 
Eq. (25) are defined by $[n=$ natural number $]$

$$
\begin{aligned}
& S_{2 n}(r) \equiv D^{-1} \int_{0}^{\infty} d k \amalg(k) k^{3} J_{2 n}(k r) \\
& \eta^{2} \equiv D^{-1} \mu \\
& \hat{\beta}_{s} \equiv D^{-1} v_{y}^{\prime} / 2, \quad \tau \equiv 2 D t,
\end{aligned}
$$

and

$$
D \equiv \pi \int_{0}^{\infty} \Pi(k) k^{3} d k
$$

where $J_{2 n}$ 's are the Bessel functions of the first kind. Equations (25) and (26) are our most general basic set of equations, and we shall now attempt their solution in various limits.

\section{Shearless System: Enstrophy Spectrum, Absolute I,evel and Shape}

In this and later sections we analyze Eq. (25) to find its steady-state solution in order to obtain steady-state turbulence levels and the associated transport. The steady states, if they exist, result from a balance of diffusion (turbulent and classical) against the gradient source (with or without the equilibrium shear). Since the system is considerably simpler without shear $\left(\widehat{\beta}_{s}=0\right)$, we begin our investigations with this case.

\section{A. Correlation function}

With $\widehat{\beta}_{s}=0$, the steady state $(\partial / \partial \tau=0) \mathrm{Eq} \cdot(25)$ allows the decomposition

$$
C(r, \varphi)=A(r)+B(r) \cos 2 \varphi
$$

with $A(r)$ and $B(r)$ obeying two independent second order differential equations

$$
L_{r} A(r) \equiv \frac{1}{r} \frac{d}{d r}\left[\eta^{2}+1-S_{0}(r)-S_{2}(r)\right] r \frac{d}{d r} A(r)=-\frac{S_{0}(r)}{L_{0}^{2}}
$$


and

$$
L_{r} B(r)-\frac{4}{r^{2}}\left[\eta^{2}+1-S_{u}(r)+S_{2}(r)\right] B(r)=-\frac{S_{2}(r)}{L_{0}^{2}} .
$$

Notice that $A(r)$ represents the angle averaged spectrum $\oint C(r, \varphi) d \varphi$, while $B(r)$ measures the anisotropy. In this section, we limit ourselves to solving for the isotropic part $A(r)$. Making use of the appropriate properties of the Bessel functions, the first integral of Eq. (28a) is trivially obtained,

$$
\frac{d A(r)}{d r}=-\frac{1}{L_{0}^{2}} \frac{S_{1}(r)}{\eta^{2}+1-2 S_{1}(r) / r}
$$

where

$$
S_{1}(r)=\int_{0}^{\infty} d k: \Pi(k) k^{2} J_{1}(k r) / \int_{0}^{\infty} \Pi(k) k^{3} d k
$$

and the determination of $A$ is reduced to quadrature. The integration constant is to be determined by demanding that $A(r) \rightarrow 0$ as $r \rightarrow \infty$. 'The boundary condition is a statement of the intuitive physical notion that two-fluid elements, when infinitely apart, are not correlated. The acceptable solution

$$
A(r)=\frac{1}{L_{0}^{2}} \int_{r}^{\infty} \frac{S_{1}^{\prime}\left(r^{\prime}\right) d r^{\prime}}{\eta^{2}+1-2 S_{1}\left(r^{\prime}\right) / r^{\prime}}
$$

though implicit $\left[S_{1}\left(r^{\prime}\right)\right.$ is itself an integral], is quite remarkably simple.

At his stage, we remind the reader that $S_{1}(r)$ can be explicitly evaluated only if we had detailed knowledge of $\Pi(k)$. In Sec. VI, we shall indeed assume a reasonable model for $\Pi(k)$ and carry out an explicit calculation not only for $A(r)$, but for the anisotropic part $B(r)$ also. In this section our attempt is to elucidate several general, model [for $\Pi(k)$ ] independent properties of $A(r)$ and its Fourier transform, the spectral function $I(k)$.

Let us first examine the function $S_{1}(r)\left[\mathrm{Eq}_{1} \cdot(30)\right]$. For any reasonable function $\Pi(k)$ which goes to zero sufficiently rapidly as $k \rightarrow \infty, S_{1}(r) \rightarrow 0$ exponentially as $r \rightarrow \infty$ because $J_{1}(k r)$ oscillates very rapidly for large $r$. For small $r$, one can expand $J_{1}(k r)$, and find that

$$
S_{1}(r)=\frac{r}{2}\left[1-\frac{\left\langle k_{1}^{2}\right\rangle}{8} r^{2}\right] \text { as } r \rightarrow 0
$$


where

$$
\left\langle k_{\perp}^{2}\right\rangle=\int_{0}^{\infty} d k k^{5} \Pi(k) / \int_{0}^{\infty} d k \Pi(k) k^{3}
$$

is the spectral average of the square of the perpendicular wavenumber. Note that Eq. (32) is meaningful if and only if the integral $\int \Pi(k) k^{5} d k$ exists, i.e., if for large $k, \Pi(k)$ goes to zero faster than $k^{-6}$. We shall assume this to be true for the problems of interest.

The limiting properties of the function $S_{1}(r)$ imply that the integrand in Eq. (31) is exponentially small for large $r$, but becomes large for small $r$. For small $r$, the denominator, $\eta^{2}+\left\langle k_{\perp}^{2}\right\rangle r^{2} / 8$, tends to be very small because the classical dissipation process (represented by $\eta^{2}$ ) is very weak for cases of practical interest. The assumption $\eta^{2} \ll 1$ is essential to our success in obtaining uniformly valid expressions for the correlation function. It is expected, then, that the principal contributions to $A(r)$ will come from the region $\left\langle k_{\perp}^{2}\right\rangle r^{2} / 8 \sim \eta^{2}$. With this realization, it becomes possible to obtain an approximate, but uniformly valid (for all $r$ ) expression for $A(r)$,

$$
A(r)=-\frac{2}{L_{0}^{2}\left\langle k_{\perp}^{2}\right\rangle} \ln \left[1-\frac{2 S_{1}(r)}{r\left(1+\eta^{2}\right)}\right]
$$

which is derived in Appendix C, and goes exponentially to zero $A(r) \rightarrow(2 / r) S_{1}(r)$ as $r$ goes to infinity. For small $r$, Eqs. (32a) and (33) yield

$$
C(r) \underset{r \rightarrow 0}{\longrightarrow}-\frac{2}{L_{0}^{2}\left\langle k_{\perp}^{2}\right\rangle} \ln \left[\frac{\eta^{2}+\left\langle k_{\perp}^{2}\right\rangle r^{2} / 8}{1+\eta^{2}}\right]
$$

indicating that $C(r \rightarrow 0)$ diverges logarithmically for the inviscid system. We shall come back to discuss the wealth of information contained in Eqs. (33)-(34).

\section{B. Spectral function, total spectral intensity}

Although the expression for the spatial correlation function $A(r)$ can be readily interpreted, it is more instructive to follow the common practice of dealing with the spectral function. The isotropic part of the spectrum (we need to solve for $B(r)$ in order to obtain the anisotropic 
part) is just the Fourier-Bessel transform of $A(r)$

$$
I^{(0)}(k) \equiv \frac{1}{2 \pi} \int_{0}^{\infty} r d r J_{0}(k r) A(r) \equiv \frac{g}{2 \pi} \int_{0}^{\infty} r d r J_{0}(k r) \ln \left[1-\frac{2 S(r)}{r\left(1+\eta^{2}\right)}\right]
$$

where $g=-2 / L_{0}^{2}\left\langle k_{\perp}^{2}\right\rangle$. Integrating by parts, and remembering that $S_{1}(r)$ goes to zero exponentially at $r=\infty$, we obtain [new integration variable $p=k r$ ]

$$
I^{(0)}(k)=-\frac{2 g}{k^{2}} \int_{0}^{\infty} \frac{p d p J_{1}(p)}{1+\eta^{2}-2(k / p) S_{1}(p / k)} \frac{\partial}{\partial p}\left[\frac{S_{1}(p / k)}{p / k}\right] .
$$

Exact evaluation of the above integral for general values of $k$ is not obvious. For small values of $k$, it is straightforward to see [from Eq. (34)] that $I^{(0)}(k)=-g\left[a_{1}-a_{2} k^{2}+\cdots\right]$ has the local parabolic form. For arbitrary values of $k$ (not very small), the principal contributions to the integral again comes from the region $p / k \ll 1$ where the denominator becomes small. For moderate values of $k$, therefore, we can approximate

$$
I^{(0)}(k)=\frac{4 g}{k^{2}} \int_{0}^{\infty} \frac{p^{2} d p J_{1}(p)}{p^{2}+8 k^{2} \eta^{2} /\left\langle k_{\perp}^{2}\right\rangle}=\frac{4 g}{k} \sqrt{\frac{8}{\left\langle k_{\perp}^{2}\right\rangle}} K_{1}\left(\sqrt{\frac{8 \eta^{2}}{\left\langle k_{\perp}^{2}\right\rangle}} k\right)
$$

where $K_{1}$ is the modified Bessel function of the second kind. To the best of our knowledge, Eq. (37) represents one of very few close form analytic expressions for the spectrum in a turbulent flow valid for a wide range of $k$; moderate to large. For the inviscid problem $\left(\eta^{2}=0\right)$, the isotropic spectral function $I^{(0)}(k) \equiv\left\langle\psi_{k}\right\rangle^{2}$ vanishes algebraically as $k^{-2}$ in the asymptotic $k$ region. This is, of course, equivalent to the correlation function $C(r) \rightarrow \ell n r$ as $r \rightarrow 0$.

The inviscid limit of our theory can be readily compared with earlier theories, in particular, with that of Kraichnan. ${ }^{29}$ We find that for the Hasegawa-Mima equation, ${ }^{19-20}$ where $\psi=\phi-\nabla^{2} \phi$ is an inviscid constant of motion, our asymptotic scaling agrees with the prediction of Kraichnan. ${ }^{29}$ This agreement (in the appropriate limit) indicates that the present correlation theory has been successful in dealing with the spectral problem when the turbulent processes dominate the classical ones. Our results, however, are quite general; the 
calculated enstrophy spectrum pertains to a wider class of theoretical models than the one represented by Hasegawa-Mima equations.

A word of caution is in order here. For multiple field systems, where the enstrophy could be a complicated function of the basic fields (for example, the Hasegawa-Wakatani turbulence model $\left.{ }^{21}\right)$, the relationship between the field energy $E(k)$ and the enstrophy could be quite different from the Hasegawa-Mima model. In such cases $\left\langle\psi_{\mathbf{k}}\right\rangle^{2} \sim k^{-2}$ would not necessarily predict Kraichnan's result of $E(k) \sim k^{-3}$.

It is also important to stress the crucial role played by viscosity in achieving a steady state. It is well known that enstrophy in the Hasegawa-Wakatani turbulence model does not saturate when viscosity is zero. The source of this problem can be traced to the weak algebraic behavior $k^{-2}$ as $k \rightarrow \infty$. Notice that, for this behavior, the large $k$ contribution makes the total spectral intensity $\mathcal{E}$ diverge. The form given by Eq. $(37)\left(\eta^{2} \neq 0\right)$, on the other hand, falls exponentially for large $k$, making the appropriate contributions to $\mathcal{E}$ finite. A computer simulation of the Hasegawa-Wakatani model can provide a strong test for the validity of Eq. (37). The total value of the spectral intensity of enstrophy

$$
\mathcal{E}=\int_{0}^{\infty} d k k I^{(0)}(k)
$$

cannot be calculated using the formula (37) because it is invalid near $k=0$. Can we, then, derive a simple formula for the integrated spectral intensity $\mathcal{E}$ ? The answer is in the affirmative. In fact, for the spatially uniform systems, there exists a kind of an optical theorem which relates the total spectral or fluctuating content to the zero distance correlation, i.e.,

$$
\left\langle\psi^{2}(0)\right\rangle=\int d \mathbf{r} C^{\prime}(\mathbf{r})=A(0)=\int d \mathbf{k} I(\mathbf{k}) \equiv \mathcal{E}
$$

which, coupled with Eq. (34) for $A(r)$, yields

$$
\mathcal{E}=-\frac{2}{L_{0}^{2}\left\langle k_{\perp}^{2}\right\rangle} \ln \frac{\eta^{2}}{1+\eta^{2}}
$$


implying that viscosity will determine the absolute intensity level of the fluctuations. For $\eta=0, \mathcal{E}$ diverges confirming the statement made in the last paragraph.

The intensity levels predicted by Eq. (39) differ from the usual mixing length estimates by the rather large logarithm factor. The differences are even deeper: one can be easily misled by the apparent simplicity of Eq. (39), and believe that it immediately yields the total spectral intensity when the strength of the classical dissipation process (viscosity) is given. This is far from the truth. In fact, the dissipation coefficient $\eta^{2}$ has been obtained by normalizing the classical viscosity $\mu$ by the 'diffusion coefficient' $D=\pi \int_{0}^{\infty} \Pi(k) k^{3} d k$, which through $\Pi(k)$ depends on $\left\langle|\phi|^{2}\right\rangle$ and hence $\mathcal{E}=\left\langle\psi^{2}(0)\right\rangle$ for self-consistency. As a result the modification to the mixing length estimates is not just a simple multiplicative factor. Investigation of this issue is the main subject of the next section.

Equation (39), yielding the absolute turbulence intensity, can be seen as the statement of a 'fluctuation dissipation' theorem ${ }^{30}$ in the current context, and is a major result of-our paper.

\section{Explicit $\mathcal{E}$, and the diffusion coefficient $D$}

A totally self-consistent derivation for $\mathcal{E}(D)$ is well nigh impossible because it would require a closure scheme for $\psi$ (we had assumed a Gaussian closure for $\phi$ and not $\psi$ ). The difficulty is partially avoided by assigning a parametric dependence of $D$

$$
D=D^{*} \mathcal{E}^{\gamma}
$$

where $D^{*}$ is an appropriate ambient diffusion coefficient (per unit intensity), and $\gamma$ is a parameter. In the weak turbulence limit, the diffusion coefficient scales with $\mathcal{E}(\gamma=1)$ while in the renormalized strong turbulence theories $\gamma$ tends to 0.5 . We shall, however, consider $\gamma$ to be a floating parameter lying between 0.5 and 1.0 , and is to be determined experimentally.

Between Ecls. (39) and (40), we can obtain a transcendental equation for either $\mathcal{E}$ or $D$ by eliminating the other. Since the classical dissipation process is weak, i.e., $\eta^{2} \ll 1$, we 
shall simplify Eq. (39) to

$$
\mathcal{E}=-\frac{2}{L_{0}^{2}\left\langle k_{\perp}^{2}\right\rangle} \ln \eta^{2}=-\frac{2}{L_{0}^{2}\left\langle k_{\perp}^{2}\right\rangle} \ln \frac{\mu}{D}
$$

which, in conjunction with $(40)$, leads to either [for $\mathcal{E}$ ]

$$
\mathcal{E}=-\frac{2}{L_{0}^{2}\left\langle k_{\perp}^{2}\right\rangle} \ln \frac{\mu}{D^{*}} \mathcal{E}^{-\gamma}
$$

or $($ for $D)$

$$
D=D^{*}\left[-\frac{2}{L_{0}^{2}\left\langle k_{\perp}^{2}\right\rangle} \ln \frac{\mu}{D}\right]^{\gamma} \text {. }
$$

These equations could be readily solved graphically. However, for small $\mu / D^{*}$ (or strong turbulence limit), simple explicit expression can be readily calculated:

$$
\begin{aligned}
& \mathcal{E}=-\frac{2}{L_{0}^{2}\left\langle k_{\perp}^{2}\right\rangle} \ln \left[\frac{\mu}{D^{*}}\left(\frac{L_{0}^{2}\left\langle k_{\perp}^{2}\right\rangle}{2}\right)^{\gamma}\right] \\
& D=D^{*}\left[-\frac{2}{L_{0}^{2}\left\langle k_{\perp}^{2}\right\rangle} \ln \left[\frac{\mu}{D^{*}}\left(\frac{L_{0}^{2}\left\langle k_{\perp}^{2}\right\rangle}{2}\right)^{\gamma}\right]\right]^{\gamma},
\end{aligned}
$$

and are determined entirely in terms of the experimentally measurable quantities $\mu, D^{*}, L_{0}^{2}$ and $\left\langle k_{\perp}^{2}\right\rangle$. Both $D$ and $\mathcal{E}$ are determined by the logarithm of the small classical viscosity $\mu$. Simplified results given in Eqs. (44) and (45) are valid only in the small dissipation, and hence in the large turbulence level limit. Results true for moderate levels of turbulence can be easily obtained by solving Eqs. (39) and (40) graphically or numerically. Equations (44) and (45) clearly reveal that larger classical dissipation would result in lower (logarithmically) fluctuation levels, and in reduced turbulent transport.

In this section we have, quite comprehensively, solved the problem of $2 \mathrm{D}$ turbulence for a viscous shearless flow. The results for inviscid flow can be derived simply by taking the $\mu\left(\eta^{2}\right) \rightarrow 0$ limit. Analytical expressions for the two-point correlation function $A(r)$, the spectral function $I^{(0)}(k)$, the total spectral intensity $\mathcal{E}$, and the effective diffusion coefficients 
$D$, are obtained. These functions should constitute a fairly complete description of the turbulence problem studied in this section.

Notice that we have not displayed solutions for $B(r)$, the anisotropic part of the spectrum. These solutions, though tedious, are quite straightforward to derive. We shall deal with them in Sec. VI.

\section{Inviscid Shear Flow}

In the preceding section, we concentrated on obtaining uniformly valid solutions for the correlation function $C(r, \varphi)$ in the relatively simpler, but theoretically extremely interesting, case of turbulent flows without velocity shear. From the correlation function, we calculated absolute turbulence levels and the associated transport coefficient. Analytical progress was possible because $C(r, \varphi)$ could be decomposed exactly into $A(r)+B(r) \cos 2 \varphi$ with $A(r)$ and $B(r)$ obeying independent equations. Such a decomposition is clearly not possible if the velocity shear is nonzero, i.e., $\beta_{s} \neq 0$ in $\mathrm{Eq}_{\mathrm{q}}$. (25). In this section we develop approximate analytical techniques to solve for the correlation function $C(r, \varphi)$, and the spectral function $I(k, \epsilon)$ for arbitrarily large but inviscid shear flows. The model independent theory, developed here, is asymptotic in nature..

\section{A. Asymptotic correlation function $C(r, \varphi)$ for arbitrary shear flow}

An examination of Eq. (26) clearly reveals that the functions $S_{2 n}(r)$ appearing in Eq. (25) have reasonably simple, model independent forms in the limit $r \rightarrow 0$. In Sec. IV we had also shown that (in the shearless case) the principal contributions to the spectrum $I^{(0)}(k)$ [for moderate to large $k$ ] carne from the short distance ( $s m a l l ~ r$ ) correlations. We shall, therefore, proceed to solve the inviscid version of $\mathrm{E}_{\mathrm{l}} .(25)\left[\eta^{2}=0\right]$ in the small $r$ limit. Expanding the Bessel functions $J_{2 n}(k r)$ in Eq. (26) for small argument, and keeping only the leading order 
terms, Eq. (25) reduces to

$$
\left\{\frac{1}{r} \frac{\partial}{\partial r} r^{3} \frac{\partial}{\partial r}+3 \frac{\partial^{2}}{\partial \varphi^{2}}-\beta_{s}\left[\frac{r}{2} \sin 2 \varphi \frac{\partial}{\partial r}+\cos ^{2} \varphi \frac{\partial}{\partial \varphi}\right]\right\} C(r, \varphi)=g
$$

where $\beta_{s}=8 \hat{\beta}_{s} /\left\langle k_{\perp}^{2}\right\rangle$ and $g=-8 / L_{0}^{2}\left\langle k_{\perp}^{2}\right\rangle$.

We start with the solution for small shear, $\beta_{s} \ll 1$. Taking the $\varphi$ average of $\mathrm{Eq}$. (46), and also its $\sin 2 \varphi, \cos 2 \varphi \ldots$ moments, the following moment equations result:

$$
\begin{gathered}
\left(\frac{r}{2} \frac{\partial}{\partial r}+1\right) \overline{(\sin (2 \varphi) C)}+\frac{1}{r} \frac{\partial}{\partial r} r^{3} \frac{\partial}{\partial r} \bar{C}=g \\
\left(\frac{1}{r} \frac{\partial}{\partial r} r^{3} \frac{\partial}{\partial r}-12\right) \overline{(\cos 2 \varphi C)}-\beta_{s}\left(\frac{r}{4} \frac{\partial}{\partial r} \overline{(\sin 4 \varphi C)}+\overline{(\sin (2 \varphi) C)}+\overline{(\sin (4 \varphi) C)}\right)=0 \\
\left(\frac{1}{r} \frac{\partial}{\partial r} r^{3} \frac{\partial}{\partial r}-12\right) \overline{(\sin (2 \varphi) C)}-\beta_{s}\left(\frac{r}{4} \frac{\partial}{\partial r}[\bar{C}-\overline{\cos (4 \varphi) C)}]-\overline{(\cos (2 \varphi) C)}+\overline{(\cos (4 \varphi) C)}\right)=0
\end{gathered}
$$

where $\bar{x}=\oint(d \varphi / 2 \pi) x$ is the angle averaged quantity. In the small shear limit, we can close the system by neglecting $4 \varphi$ and higher moments, and find that the angle averaged part $\bar{C}$ satisfies

$$
\frac{\beta_{s}^{2}}{4\left(12+\beta_{s}^{2} / 12\right)}\left(1+\frac{r}{2} \frac{\partial}{\partial r}\right) r \frac{\partial}{\partial r} \bar{C}+\frac{1}{r} \frac{\partial}{\partial r} r^{3} \frac{\partial}{\partial r} \bar{C}=g,
$$

with the solution

$$
\bar{C}=\frac{g}{2+\frac{\beta_{s}^{2}}{4\left(12+\beta_{s}^{2} / 12\right)}} \cdot \ln r
$$

which reduces to $\bar{C}=(g / 2) \ln r$ in the limit $\beta_{s}=0$. Equation (51) clearly shows that as $\beta_{s}$ increases, the relative turbulence level [measured by $\bar{C}$ ] is suppressed.

Although the approximate result for small shear [Eq. (51)] is quite revealing, it is worthwhile (and possible) to solve Eq. (46) exactly for arbitrary shear. The procedure consists of assuming a solution of the form

$$
C(r, \varphi)=\Omega \ln r+x(\varphi)
$$


where $X(\varphi)$ is a function of $\varphi$ alone, and the eigenvalue $\Omega$ is to be determined by imposing periodicity constraint on $\chi_{(\nu)}$. Substituting $\mathrm{Eq}_{\mathrm{q}}$. (52) into (46) yields $\left[\zeta \equiv 2 \varphi, b_{s} \equiv \beta_{s} / 12\right]$

$$
\frac{\partial^{2} \chi}{\partial \zeta^{2}}-b_{s}(1+\cos \zeta) \frac{\partial \chi}{\partial \zeta}=\frac{g-2 \Omega}{12}+\frac{b_{s} \Omega}{3} \sin \zeta,
$$

which is integrated once to obtain the explicitly periodic function

$$
\frac{\partial \chi}{\partial \rho}=e^{t s \sin \zeta} \sum_{n=-\infty}^{\infty} T_{n}(\Omega)\left[n \sin n(\zeta+\pi / 2)-b_{s} \cos n(\zeta+\pi / 2)\right]
$$

where

$$
T_{n}\left(b_{s}, \Omega\right)=\frac{(g-2 \Omega) I_{n}\left(b_{s}\right)-6 b_{s} \Omega I_{n}^{\prime}\left(b_{s}\right)}{2\left(b_{s}^{2}+\eta^{2}\right)},
$$

$I_{n}\left(b_{s}\right)$ is the modified Bessel function of the first kind, and $I_{n}^{\prime}$ is the derivative of $I_{n}$ with respect to its argument. Further integration of Eq. (54) is straightforward. It, however, results in the explicitly non-periodic term (linear in $\zeta$ )

$$
-b_{s} \sum_{n=-\infty}^{+\infty}(-1)^{n} T_{n}\left(b_{s}, \Omega\right) I_{n}\left(b_{s}\right) \zeta \equiv F\left(b_{s}, \Omega\right) \zeta .
$$

Thus, we obtain the eigenvalue $\Omega$ (which is really a measure of the intensity of turbulence) by imposing the periodicity constraint

$$
F\left(b_{s}, \Omega\right)=0
$$

which leads to

$$
\Omega=\frac{g}{2}\left[1+3 b_{s} \frac{\sum_{n=-\infty}^{+\infty}(-1)^{n} \frac{I_{n}\left(b_{s}\right) I_{n}^{\prime}\left(b_{s}\right)}{n^{2}+b_{s}^{2}}}{\sum_{n=-\infty}^{+\infty}(-1)^{n} \frac{I_{n}^{2}\left(b_{s}\right)}{n^{2}+b_{s}^{2}}}\right]^{-1} \equiv \frac{g}{2} S_{p}\left(b_{s}\right),
$$

a rather complicated function of the shear parameter. Notice that the coefficient of $(g / 2) \ell n r$ in the expression for the $\overline{C^{\prime}(r, \nu)}\left[\mathrm{Eq}_{\mathrm{q}}\right.$. (52)-(57) or Eq. (51) for the weak shear case] can be naturally called the suppression factor, and is a quantity of great interest to the experiments studying the change of fluctuation levels with the strength of the shear flow. In Fig. 1a, we 
plot $S_{p}\left(b_{s}\right)$ as a function of $\hat{v}_{y}^{\prime} \equiv v_{y}^{\prime} t_{c}$, where $t_{c} \equiv\left[2\left\langle k_{\lrcorner}^{2}\right\rangle D\right]^{-1}$ is the decorrelation time [the time it takes for two-fluid elements to become decorrelated], and the argument $b_{s}=(2 / 3) \hat{v}_{y}^{\prime}$. The solid line represents the exact function $S_{p}$, while the dotted-dashed line is the asymptotic value proportional to $\left(\hat{v}_{y}^{\prime}\right)^{-2 / 3}$.

We must again warn the reader that the function $S_{p}$ does not completely and explicitly characterize the true suppression of turbulence, because the shear parameter $\hat{v}_{y}^{\prime}\left(b_{s}\right)$ is a functional of the turbulence through the agency of the diffusion coefficient $D$ [see Sec. IV.C]. To take this into consideration, let us again assume that $D$ scales as $\left\langle|\psi|^{2}\right\rangle^{\gamma}$, i.e., $D=D_{0}\left\langle|\psi|^{2}\right\rangle^{\gamma} /\left\langle|\psi|^{2}\right\rangle_{0}^{\gamma}$, where $D\left(D_{0}\right.$ and $\left.\left\langle|\psi|^{2}\right\rangle,\left\langle|\psi|^{2}\right\rangle_{0}\right)$ respectively denote the diffusion coefficient and the turbulence levels with (without) velocity shear. It is now convenient to define the turbulence suppression by the nonlinear equation

$$
\xi=S_{p}\left[\frac{2}{3} \frac{\hat{v}_{y 0}^{\prime}}{\xi^{\gamma}}\right]
$$

where $\xi \equiv\left\langle k_{\perp}^{2}\right\rangle\left\langle|\psi|^{2}\right\rangle /\left(\left\langle k_{\perp}^{2}\right\rangle\left\langle|\psi|^{2}\right\rangle\right)_{0}, \hat{v}_{y 0}^{\prime} \equiv v_{y}^{\prime} t_{c 0} /\left[\left\langle k_{\perp}^{2}\right\rangle /\left\langle k_{\perp}^{2}\right\rangle_{0}\right]^{1-\gamma}, t_{c 0}=\left(2\left\langle k_{\perp}^{2}\right\rangle D\right)_{0}^{-1}$ [note that in Ref. 16, $t_{c 0}$ is defined as $\left.\left(\sqrt{2}\left\langle k_{\perp}^{2}\right\rangle D\right)_{0}^{-1}\right]$, and the subscript zero denotes the quantities without shear flow. The numerical solutions of Eq. (58), i.e., $\xi=\xi\left(\widehat{v}_{y 0}^{\prime}\right)$ are plotted in Fig. 1b as the solid curves; the dashed curves in Figs. 1a-1b are similar results from the orbit theory (Heisenberg picture). The resuits from the two approaches will be compared and contrasted in Sec. VII.

To complete the picture, the periodic function $\chi(\varphi)$, representing the anisotropic part (an essential consequence of the shear flow), is given by

$$
\chi(\varphi)=\sum_{m \neq n=-\infty}^{\infty} \frac{T_{n}\left(b_{s}, \Omega\right) I_{m}\left(b_{s}\right)}{m-n}\left[b_{s} \sin \alpha_{m, n}+n \cos \alpha_{m, n}\right]
$$

where $\alpha_{m, n}=2(n-m) \varphi+(n+m) \pi / 2$. To visualize the angular distribution of the correlation function $C(r, \varphi)[=\Omega \ln r+\chi(\varphi)]$, we draw contour plots for several values of the shear parameter and for a fixed small value of $r=r_{0}$ with $\ell n r_{0}=-1$. It can be seen from 
Figs. $2 \mathrm{a}-2 \mathrm{~b}$ that for sufficiently large shear flow the correlation length in the $y(x)$ directions becomes considerably longer (shorter).

\section{B. Wavenumber space, integral equation, and the asymptotic spectral function}

In this paper we have and we shall concentrate primarily on solving the various aspects of the $2 \mathrm{D}$ turbulence problem in real $(r, \varphi)$ space. It is possible, however, to derive an equivalent of Eq. (25) in the wavenumber space which determines the behavior of the Fourier transformed spectral function $I(\mathbf{k})$ related to $C(\mathbf{r})$ by

$$
I(\mathbf{k})=\int d \mathbf{r} e^{i \mathbf{k} \cdot \mathbf{r}} C(\mathbf{r})
$$

Taking the Fourier transform of Eq. (24), one obtains the spectrum equation $\left[k_{x}=k \cos \epsilon, k_{y}=\right.$ $k \sin \epsilon]$

$$
\begin{gathered}
\left(\frac{\partial}{\partial t}-v_{y}^{\prime} k_{y} \frac{\partial}{\partial k}+2 \mu k^{2}\right) I(\mathbf{k})-2 \int d \mathbf{k}^{\prime}\left[\mathbf{b} \times \mathbf{k}^{\prime} \cdot \mathbf{k}\right]^{2} \Pi\left(\mathbf{k}^{\prime}\right) \\
\times\left[I\left(\mathbf{k}+\mathbf{k}^{\prime}\right)-I(\mathbf{k})\right]=2 \frac{k_{y}^{2}}{L_{0}^{2}} \Pi(\mathbf{k})
\end{gathered}
$$

which is an integrodifferential equation, and is, in general, difficult to handle. In the large $k$ limit, however, this complicated equation can be simplified to a solvable differential equation. By making use of the fact that for $k \rightarrow \infty$,

$$
I\left(\mathbf{k}+\mathbf{k}^{\prime}\right)-I(\mathbf{k}) \simeq k_{\mu}^{\prime} \frac{\partial I}{\partial k_{\mu}}+\frac{1}{2} k_{\mu}^{\prime} k_{\nu}^{\prime} \frac{\partial^{2} I}{\partial k_{\mu} \partial k_{\nu}}
$$

Eq. (59) (to the leading order) reduces to

$$
\begin{gathered}
\beta_{s}\left[\frac{k}{2} \sin 2 \epsilon \frac{\partial}{\partial k}-\sin ^{2} \epsilon \frac{\partial}{\partial \epsilon}\right] I(k, \epsilon) \\
+\frac{8 \eta^{2}}{\left\langle k_{\perp}^{2}\right\rangle} k^{2} I(k, \epsilon)+\left[\frac{1}{k} \frac{\partial}{\partial k} k^{3} \frac{\partial}{\partial k}+3 \frac{\partial^{2}}{\partial \epsilon^{2}}\right] I(k, \epsilon)=0
\end{gathered}
$$


which, in the inviscid range, can be solved for periodic solutions exactly in the manner adopted in Sec. V.A. Notice that for $\beta_{s}=0, I(k, \epsilon) \sim I^{(0)}(k)$, and Eq. (62) allows exactly the modified Bessel function solution $I^{(0)}(k) \sim k^{-1} K_{1}\left(\sqrt{8 \eta^{2} /\left\langle k_{\perp}^{2}\right\rangle} k\right)$.

For the inviscid shear problem, the substitution $I(k, \epsilon) \equiv k^{-2} Q(\epsilon)$ leads to the manifestly periodic solution

$$
Q(\epsilon)=e^{-b_{s} \sin 2 \epsilon}\left\{I_{0}\left(b_{s}\right)+2 b_{s} \sum_{n=1}^{\infty} \frac{I_{n}\left(b_{s}\right)}{n^{2}+b_{s}^{2}}\left[b_{s} \cos 2 n(\epsilon-\pi / 4)-n \sin 2 n(\epsilon-\pi / 4)\right]\right\}
$$

where $I_{n}$ 's are again the modified Bessel function of the first kind. The angular spectrum of enstrophy for various values of the shear parameter is displayed as a contour plot in Fig. 3. The amplitude is normalized to unity at $\epsilon=0$. Starting from an isotropic form for $\widehat{v}_{y}^{\prime}=0$ [curve a], the spectrum stretches to a dumbbell shape in response to the increasing shear [curves $b, c, d]$ getting more elongated in the $k_{x}[k \cos \epsilon]$ direction implying that the correlation length along $x$ becomes shorter than the correlation length along $y$.

\section{Global Solutions for a Model $\Pi(k)$}

In Sec. IV we had obtained, in the shearless case, approximate model independent uniformly valid solutions for the correlation function $C(\mathbf{r})$ from which analytical expressions for the absolute turbulence level and the diffusion coefficient were calculated. We noted in Sec. V that the complications brought about by the velocity shear forced us to solve the problem only in the asymptotic $[r \rightarrow 0$ for $C(\mathbf{r})$, and $k \rightarrow \infty$ for $I(\mathbf{k})]$ limits. The asymptotic solutions did lead us to the extremely important result that the presence of velocity shear tends to suppress the turbulent enstrophy levels. These results, though reasonable, could be criticized on the ground that we have demonstrated the turbulent suppression only in the asymptotic range, and not in the entire range of turbulence. The objection would have been particularly serious if we had depended on the result of Sec. V.B [large $k$ limit for $I(\mathbf{k})$ ] alone because most of the spectral energy, perhaps, resides in the low to moderate range of $k$. 
This objection is somewhat mitigated because we rely much more on the finding of Sec. V.A, where the short distance correlation function is solved for arbitrary shear. Although this is also an asymptotic $(r \rightarrow 0)$ theory, it is a much better qualitative index of the overall turbulence, because the principal contribution to the spectral function [Fourier transforms of $C(\mathbf{r})$ ], for a broad range of $k$, indeed comes from short distance correlations; the long distance correlations tend to be small.

In order to place our conjecture on a firmer footing, we shall have to do a global calculation with $\beta_{s} \neq 0$. Settling this point has much more than mere academic interest; it is essential to know the effects of shear flow on the entire range of turbulent spectrum, if we have to build a transport theory of experimental interest.

Working towards a global solution of the full Eq. (25) requires that we know the functional forms for $S_{2 n}(r)$. This is possible only if we know the functional form of $\Pi(k)$, which in fact depends on the very solutions we seek. Notice that in the previous sections, we could mechanically carry out calculations without specifying $\Pi(k)$, and only in the end we had to face the problem of self-consistency which we solved by proposing relations of the type Eq. (40).

What form(s) do we choose for $\Pi(k)$ ? Naturally, $\Pi(k)$ must go to zero reasonably fast as $k \rightarrow \infty$. Our experience in the shearless case, as well as the asymptotic sheared flow case, suggests that $\Pi(k)$ must fall faster than $k^{-6}$ as $k \rightarrow \infty$ because only then integrals of the type $\int_{0}^{\infty} \Pi(k) k^{5} d k$ can exist. It turns out that the system [Eq. 25] is quite robust, and reasonable choices of $\Pi(k)$ lead to consistent qualitatively as well as quantitatively similar results. Numerical solutions of $\mathrm{Eq}$. (25) for several models for $\Pi(k)$ [Gaussian, exponential, Lorentzian], with appropriate normalizations, lead to results which are barely distinguishable in their essential characteristics. As a result, we present here the simplest model for which $S_{2 n}(r)$ become elementary functions. The model is a Gaussian

$$
\Pi(k)=\Pi_{0} e^{-\lambda k^{2}}
$$


with two parameters $\lambda$ and $\Pi_{0}$ measuring respectively the width and the height of the spectrum. Clearly $\lambda$ needs to be identified with $2 /\left\langle k_{\perp}^{2}\right\rangle$, while $\Pi_{0}$ is related to the decorrelation time $t_{c}$ defined earlier $\left[\lambda^{3} / 2 \pi t_{c}\right]$. Using (64) to evaluate $S_{2 n}(r)$, Eq. (25) takes the explicit form $\left(\rho^{2}=r^{2} / 4 \lambda\right)$,

$$
\begin{aligned}
& \frac{1}{\rho} \frac{d}{d \rho} \rho\left[1+\eta^{2}-e^{-\rho^{2}}\right] \frac{\partial C}{\partial \rho}+\frac{1}{\rho^{2}}\left[1+\eta^{2}-e^{-\rho^{2}}\left(1-2 \rho^{2}\right)\right] \frac{\partial^{2} C}{\partial \varphi^{2}} \\
& -\beta_{s}\left[\frac{\rho}{2} \sin 2 \varphi \frac{\partial}{\partial \rho}+\cos ^{2} \varphi \frac{\partial}{\partial \varphi}\right] C=g e^{-\rho^{2}}\left[1-2 \rho^{2} \sin ^{2} \varphi\right] .
\end{aligned}
$$

For $\rho<1$, i.e., when the inverse correlation length is greater than the averaged wavenumbers, Eq. (65) could be solved by the methodology of Sec. IV. In this particular case, however, the solution for $A(\rho)$ can be obtained exactly,

$$
A(\rho)=-\frac{2}{L_{0}^{2}\left\langle k_{\perp}^{2}\right\rangle} \ln \left[1-\frac{e^{-\rho^{2}}}{1+\eta^{2}}\right]
$$

and is precisely the same as the approximate solution Eq. (33) $\left[(2 / r) S_{1}(r)=e^{-\rho^{2}}\right.$ for $\Pi(k)=$ $\Pi_{0} e^{-\lambda k^{2}}$. Thus for the model $\Pi(k)$ given by Eq. (64), Eq. (33) would be an exact uniformly valid solution. This clearly strengthens our belief in the validity of the approximations made in Sec. IV. Remember that in deriving Eq. (33), we had used the fact that small distance $(\rho<1$, in the present context) contribution to the integral [Eq. (31)] was dominant. The complete equality of this approximation with the exact model dependent solution provide a strong justification for the soundness of the turbulent suppression results derived in Sec. V.A.

The finite shear problem, represented by Eq. (65), is still a complicated partial differential equation, in which the various $(2 \varphi)$ harmonics are coupled together by the shear term. When the strength of the shear term is not very large, typical $\widehat{v}_{y}^{\prime} \lesssim 1$ or $\beta_{s}<8$, the system could be truncated, i.e., we could approximate the solution by

$$
C(\rho, \varphi) \simeq A(\rho)+B_{c}(\rho) \cos 2 \varphi+\operatorname{sgn}\left(\beta_{s}\right) B_{s}(\rho) \sin 2 \varphi+\cdots
$$

where terms proportional to $\sin 2 n \varphi(\cos 2 n \varphi)$ for $n \geq 2$ have been neglected, and where 
$\operatorname{sgn}\left(\beta_{s}\right)$ guarantees the symmetry of the system under the simultaneous transformation $\varphi \rightarrow$ $-\varphi, \beta_{s} \rightarrow-\beta_{s}$. From now on, without any loss of generality, $\beta_{s}$ is taken to be positive.

With our truncation scheme, substitution of $\mathrm{Eq}$. (67) into (65) leads to closed set of coupled equations for $A(\rho), B_{c}(\rho)$, and $B_{s}(\rho)$

$$
\begin{aligned}
& \frac{1}{\rho} \frac{d}{d \rho} \rho\left(1+\eta^{2}-\exp \left(-\rho^{2}\right)\right) \frac{d}{d \rho} A-\beta_{s}\left(\frac{\rho}{4} \frac{d B_{s}}{d \rho}+\frac{B_{s}}{2}\right)=g \exp \left(-\rho^{2}\right)\left(1-\rho^{2}\right), \\
& \frac{1}{\rho} \frac{d}{d \rho} \rho\left(1+\eta^{2}-\exp \left(-\rho^{2}\right)\right) \frac{d}{d \rho} B_{c}-\frac{4}{\rho^{2}}\left[1+\eta^{2}-\exp \left(-\rho^{2}\right)\left(1-2 \rho^{2}\right)\right] B_{c}-\beta_{s} B_{s} \\
& =g \rho^{2} \exp \left(-\rho^{2}\right) \text {, } \\
& \frac{1}{\rho} \frac{d}{d \rho} \rho\left(1+\eta^{2}-\exp \left(-\rho^{2}\right)\right) \frac{d}{d \rho} B_{s}-\frac{4}{\rho^{2}}\left[1+\eta^{2}-\exp \left(-\rho^{2}\right)\left(1-2 \rho^{2}\right)\right] B_{s} \\
& -\beta_{s}\left(\frac{\rho}{2} \frac{d A}{d \rho}-B_{c}\right)=0 .
\end{aligned}
$$

The set of Eqs. (68)-(70) is solved numerically with decaying boundary conditions for $\rho \rightarrow \infty$, and also imposing $B_{c}(\rho=0)=0=B_{s}(\rho=0),\left.(d A / d \rho)\right|_{\rho=0}=0$ at $\rho=0$. The latter constitute a consistent set, and are obtained by solving the system near $\rho=0$. The functions $A, B_{c}(\rho)$ and $B_{s}(\rho)$ are plotted as functions of $\rho$ for several values of the shear parameter $\hat{v}_{y}^{\prime}=0,0.2$ and $0.5\left[\beta_{s}=0,1.6,4.0\right]$ in Fig. $4 a-4 c$. For all these cases, $g=-1$, and $\eta^{2}=0.01$. The magnitudes of both $A(\rho)$ and $B_{c}(\rho)$ [the components present in the absence of shear] are suppressed in the entire range of $\rho$, the overall suppression increasing with increasing shear. As expected, the shear generated part $B_{s}$ [the coefficient of $\sin 2 \varphi$ term] scales with the shear parameter $\hat{v}_{y}^{\prime}$.

It can be observed from Figs. 4a-4c, that the angle averaged part $A(\rho)$ dominates other components indicating that our truncation scheme is quite good up to $\widehat{v}_{y}^{\prime}=0.5$. A much greater value of the $\hat{v}_{y}^{\prime}$ will make $B_{s}(\rho)$ comparable to $A(\rho)$ in some regions implying that the neglected coupling to higher harmonic terms may become important. In such a case, the solutions based on Eqs. (68)-(70) may no longer be quantitatively reliable. Therefore, the 
current approach is good only for weak shear.

The spectrum $I(\mathbf{k})$ follows from the solutions of correlation function through the FourierBessel transform. The three component Ansatz, Eq. (67), implies a three-component spectrum:

$$
I(k, \epsilon)=I^{(0)}(k)-I_{c}(k) \cos 2 \epsilon-I_{s}(k) \sin 2 \epsilon
$$

with

$$
\begin{aligned}
& I^{(0)}(k) \equiv \frac{1}{2 \pi} \int_{0}^{\infty} \operatorname{drr} A(r) J_{0}(k r), \\
& I_{c}(k) \equiv \frac{1}{2 \pi} \int_{0}^{\infty} \operatorname{drr} B_{c}(r) J_{2}(k r), \\
& I_{s}(k) \equiv \frac{1}{2 \pi} \int_{0}^{\infty} \operatorname{drr} B_{s}(r) J_{2}(k r),
\end{aligned}
$$

where $J_{0}, J_{2}$ are the zeroth and second order Bessel function of the first kind. Computed numerical expressions for $I^{(0)}(k), I_{c}(k)$ and $I_{s}(k)$ are shown in Figs. 5a-5c. In Fig. 5a the azimuthally averaged spectra $I^{(0)}$ are represented by solid curves $a, b$, and $c$ corresponding to different values of shear. The behavior of $I^{(0)}$ clearly indicates that the shear flow suppression (on the zeroth harmonic component) is more significant for long wavelengths than for short wavelengths. The obvious implication is that the velocity shear tends to shift the turbulent spectrum towards shorter wavelengths.

To compare these global results with those from the asymptotic theory of preceding sections, we may turn to the isotropic spectrum contained in Eq. (50). Notice that the simple logarithmic function, $\ln r$ in $\mathrm{Eq}_{\mathrm{l}}$. (50), should be replaced by the global behavior given by $\mathrm{Eq}$. (33) [Eq. (66)] to guarantee its positiveness on the whole range, i.e., the asymptotic spectrum $I_{\text {asy }}^{(0)}(k)$ can be calculated from the correlation function Eq. (33) [Eq. (66)] with 
asymptotic suppression factor,

$$
A_{\text {asy }}(\rho)=\frac{g}{4+\frac{\beta_{s}^{2}}{24+\beta_{s}^{2} / 6}} \cdot \ln \left[1-\frac{e^{-\rho^{2}}}{1+\eta^{2}}\right],
$$

by iaking the Fourier-Bessel transform. Without the replacement of $\ell n r$ by the globally valid expression, the integral defining $I_{\text {asy }}^{(0)}$ would become ill-behaved.

For several values of shear $\left[\hat{v}_{y}^{\prime}=0.2\right.$ and 0.5$]$, the numerically computed values of $I_{\text {asy }}^{(0)}$ are shown in Fig. 5a by the dotted-dashed curves $b^{\prime}$ and $c^{\prime}$ respectively. This comparison with the global values (solid curves $a, b$ and $c$ ) reveals an additional suppression occurring at longe: wavelengths in the global theory.

This additional feature has interesting consequences for ascertaining the effects of velocity shear on plasma confinement (say in the edge region of a tokarnak). Not only is the confinement improved due to a reduction in the fluctuation level (less anomalous transport), but the fluctuation spectrum, itself, is shifted towards the less dangerous shorter wavelength domain (the longer wavelengths destroy the confinement more effectively).

The spectral shift could be easily incorporated into the general scenario by numerically calculating the effective $\overline{k_{\perp}^{2}} \equiv \int_{0}^{\infty} d k k^{2} I^{(0)}(k) / \int_{0}^{\infty} d k I^{(0)}(k)$ for finite shear. In Fig. 6 we plot $\overline{k_{\perp}^{2}}$ as a function of shear for the parameters of Figs. 5a-5c. The ratio of $\overline{k_{\perp}^{2}}$ with or without shear can then be substituted into Eqss. (53)-(44) to account for the shear-induced spectral shift.

\section{Comparison with the Orbit Picture}

The bulk of this paper is devoted to the development of the correlation theory and our attempts to analytically as well as numerically solve for the two-point correlation function $C(\mathbf{r})$. A detailed knowledge of $C(\mathbf{r})$, in some sense, represents a complete knowledge of the physically interesting aspects of turbulence. We had also remarked in Sec. II, that an alternative simpler program, i.e., of developing an orbit theory could also be carried out. 
This, in fact, was done (for the nondissipative case) amongst others in Ref. 16. We are now ready to compare and contrast the two approaches.

The relative simplicity of the orbit picture is gained at the expense of limiting its range of validity. The 'closed' set of equations for the relative orbit correlation [Eqs. (1) of Ref. 16] are obtained by using the asymptotic (short distance) limits of the turbulent diffusion coefficients $D^{\mu \nu}$. When the product $\mathbf{k} \cdot \mathbf{r}$ is of order unity, higher moments of the orbit correlations will couple to the system, rendering the closure scheme invalid. Thus the orbit picture does not reflect the correct solution over the entire wavenumber range.

There is, in fact, a further problem with the orbit theory. The primary 'solution' in the orbit picture is just an equation for the decorrelation time defined to be the $e$-folding time for the relative separation of the two-fluid elements. Surely, this information says nothing about the turbulence levels. Thus one has to go back to the equation for the crrrelation function and infer from it a definition (on purely dimensional grounds) of the correlation time in terms of the fluctuation levels, i.e.,

$$
t_{c}^{-1}\left\langle|\psi|^{2}\right\rangle=\frac{2 D}{L_{0}^{2}}
$$

where it was speculated that the shear effects could be qualitatively modified by dissipation processes. Since $\mathrm{Eq}_{\mathrm{l}}$. (76) is purely schematic, i.e., the entire operator on the correlation function [for example, see Eq. (24)] is replaced by a symbol $t_{c}^{-1}$, it is only qualitatively meaningful. In order to obtain quantitatively meaningful results, one must indeed solve Eq. (24).

It turns out that there mist indeed be a numerically factor of four on the right-hand side of Eq. (76) in order to make the preceding expression of $t_{c}$ coincide with the definition given earlier in this paper. The numerical factor, however, does not affect the results of Ref. 16 concerning the relative change of turbulence amplitude under the shear flow. However, the interpolated formula [Eq. (9)] of Ref. 16 should be corrected to [suppressing the logarithmic 
multiplier]

$$
\left\langle|\psi|^{2}\right\rangle=\frac{4}{\left\langle k_{\perp}^{2}\right\rangle L_{0}^{2}+\left(\frac{v_{y}^{\prime} L_{0}^{2}}{2 D^{*}}\right)^{2 \gamma}\left(\left\langle k_{\perp}^{2}\right\rangle L_{0}^{2}\right)^{\gamma}}
$$

where $D^{*}$ is defined by $D=D^{*}\left\langle|\psi|^{2}\right\rangle^{\gamma}$.

After having placed the orbit picture in its proper perspective, we compare the results of the orbit theory with the asymptotic theory of Sec. V.A. In Fig. 1a, along with the suppression factor [Eq. (57)]

$$
S_{p}\left(b_{s}\right)=\left[1+3 b_{s} \frac{\sum_{n=-\infty}^{+\infty} \frac{(-1)^{n} I_{n}\left(b_{s}\right) I_{n}^{\prime}\left(b_{s}\right)}{n^{2}+b_{s}^{2}}}{\sum_{n=-\infty}^{+\infty} \frac{(-1)^{n} I_{n}^{2}\left(b_{s}\right)}{n^{2}+b_{s}^{2}}}\right]^{-1}
$$

plotted as the solid curve, we also have the dashed curve representing the solution of (positive root) the cubic

$$
\left[\frac{1}{S_{p}^{(O)}\left(b_{s}\right)}-1\right]\left[\frac{1}{S_{p}^{(O)}\left(b_{s}\right)}+\frac{1}{2}\right]^{2}=\frac{2}{3} b_{s}^{2}
$$

obtained in the orbit theory [the superscript $(O)$ denotes the orbit theory]. It is entirely remarkable that the two asymptotic solutions $S_{p}$ and $S_{p}^{(O)}$ agree so well [see Fig. 1a, 1b]. This agreement is quite baffling because $S_{p}\left(b_{s}\right)$ was derived in a steady-state theory while $S_{p}^{(O)}\left(b_{s}\right)$ is the result from a time evolution equation [see Ref. 16]; we are not aware of any transformation which might connect the Schrödinger picture [correlation function approach] with the Heisenberg picture (orbit theory).

\section{Summary and Discussion}

The problems of plasma turbulence (a determination of its absolute level and spectrum) are, in general, quite difficult and complicated. Satisfactory theoretical calculations of turbulent transport relevant to experiments (for example, tokamaks), therefore, are few in number. In this paper, we have made attempts to develop and solve a reasonably comprehensive model 
for plasma turbulence. The success of this program is essentially a result of the following basic assumptions:

(a) We have introduced a relatively simple 2D theoretical model for electrostatic turbulence, based on the continuity equation and the equation for quasineutrality. The system is simplified by neglecting the ion sound effect, the toroidal effects (including trapped ions, and parallel viscosity). The mechanism leading to the saturation of turbulence from the ion perpendicular viscosity and the classical diffusion. Notice that this theoretical model is still quite general; it pertains to a variety of parallel Ohm's laws describing electron dynamics.

(b) The relative simplicity of this physical model allows the development of an equal time correlation theory; the Green functions of the theory can be rigorously calculated. Naturally, the equal time theory is much more accessible because it avoids the problems associated with the frequency domain. By the same token, this theory sheds no light on the frequency spectrum of the turbulence.

(c) The principal conceptual assumption is in invoking the Gaussian process for the stochastic 'external' field $\phi$; while the reactive field (generalized enstrophy $\psi$ ) is not necessarily Gaussian. Naturally, there is no reason to believe that the electrostatic potential would be more Gaussian than the generalized enstrophy. In fact, for a physical model like the Hasegawa-Mima equation, the enstrophy $\psi=\phi-\nabla^{2} \phi$ is fully determined by $\phi$. This obvious contradiction, however, may not be as serious as it appears at first sight. The asymptotic limit of our spectrum for the Hasegawa-Mima equation [Sec. IV, $\left|\psi_{k}\right|^{2} \sim k^{-2}$ ] is found to be identical with the result obtained by Kraichnan's method ${ }^{29}$ which does not invoke Gaussian processes. One may still object that a Gaussian procsss may not pertain for the longer wavelengths, a coherent structure with large coherence length may provide an example where this assumption will not be valid. We naturally, do not consider such processes; our framework is based on spatial homogeneity and rules out coherent structures.

From an experimental point of view, it seems that our assumptions may be reasonably 
good. The measured non-Ciaussian part of plasma turbulence on the TEX'T edge plasma (for example) is found to be less than fifteen percent of the total turbulence. ${ }^{31}$

In terms of the renormalized perturbation theories, ${ }^{32-38}$ the non-Ciaussian processes are related to the three wave interactions. These processes do not appear explicitly to be a part of our theory. The three wave process is found to be important for the frequency broadening at a given wavenumber as well as the well-known spectral cascadings. The correlation theory, developed here, deals with frequency integrated correlation functions. As stated earlier, details about the frequency domain are not accessible to this theory. The spectral cascadings are generally supposed to be responsible for achieving the spectral saturation, which we have been seeking for in this paper. Therefore, one must be cautious to the terminology of 'Gaussian approximation', which should not be confused with the term used in such theories with a time scale comparable to the wave frequencies, as the renormalized perturbation theories. ${ }^{32-38}$

(d) The next basic assumption is related to the problem of self-consistency of the correlation theory. This theory is constructed as an extension of the Langevin approach for the Brownian motion to the continuous media, the reactive field is seen as a Brownian particle, whereas the active field (here the electrostatic potential) behaves like the surrounding molecules acting on the Browning particle. The self-consistency will pertain if the Brownian particles were treated as 'test particles'. In lieu of invoking the other equation for density and potential to close the system, the self-consistency adopted in the correlation theory for the reactive field is 'imposed' by some physically reasonable conditions. In this paper, a simple closure scheme, consisting of a physically interesting functional form relating the electrostatic potential ( $D$ is a functional of $\phi$ ) and the generalized enstrophy [Eq. (40)] is used. This simple closure scheme implies that the characteristic absolute turbulence level and the transport coefficient can now be approximated by the enstrophy transport through a power law. Noticeably, the basic results of the paper are independent of the linear growth rate 
of the waves, even of the nature of the linear destabilizing mechanisms. The details of the electron dynamics, which would be crucial to the destabilization mechanism, are somewhere buried in this assumed form. Naturally, the above approach does not provide a complete solution; it leaves many unanswered questions such as the frequency spectrum, determination of $\gamma$, and the individual density and potential spectrum. However, this approach seems to be reliable and satisfactory in that $\gamma$ is believed to vary only in a narrow range $(0.5>\gamma>1.0)$; and the results, e.g., these explicitly given in Sec. IV depend rather weakly on $\gamma$.

The correlation theory also assumes a separation between two length scales: the equilibrium scale is much greater than the correlation length. Unfortunately, this is generally not true at the shear layer of tokamaks, i.e., the shear flow layer width is found typically to be comparable with the radial correlation length. As a result, the shear suppression within a scale smaller than the correlation length can not be properly described by the present theory.

The technical approximation in evaluating the global shear effect [Eq. (64)] may be sufficiently satisfactory. This two-parameter ansatz can be improved further by introducing more parameters for the 'external spectrum' in both the amplitude and the shape, but this is done only at the expense of simplicity and elegance. One may verify the appropriateness of the approximations a posteriori from the obtained results. In this paper the spectrum of the inviscid generalized enstrophy $\sim 1 / k^{2}$ at short wavelengths. For the Hasegawa-Mima case $^{19-20}\left|\psi_{k}\right|^{2}=k^{4}|\phi|^{2}$ as $k \rightarrow \infty$, would imply, along with the definition of $\Pi(k)$ [see Appendix A] that $\Pi(k) \sim k^{-8}$, which indeed will yield $\left|\psi_{k}\right|^{2} \sim 1 / k^{2}$. It should be noticed that the spectral shape of $\Pi(k)$ is unimportant in the small shear limit, as long as the dissipation is very small $(\eta \ll|\ell n \eta|)$. We expect that this holds also for the moderate shear cause it is only the integrals of the spectrum that are relevant to the theory, and the integrals are quite insensitive to the detailed shapes. Therefore, the back-reac lion of the deformed spectrum due to the shear flow on the presumably fixed 'external field' spectrum can be neglected as a higher order effect. 
Having given a lengthy critique of our physical model and theoretical framework, we now summarize the principal results of this paper: (1) We obtained analytical solutions for the correlation function, the spectral function, and the total turbulent intensity pertaining to a shearless viscous flow, and demonstrated that the total turbulent intensity is simply related to the classical dissipation (viscosity) implying a kind of fluctuation-dissipation theorem, (2) for the sheared flow, we obtained approximate analytical and numerical solutions in a variety of ways including a numerical model dependent global theory for weak shear, and an inviscid asymptotic, model independent theory for arbitrary shear. The results of these approaches were in general quantitative agreement in overlapping domains. All approaches led to the suppression of turbulence by the velocity shear; the global theory, in addition, predicts a shear-induced spectral shift to less dangerous short wavelengths. Thus the shear effects improve plasma confinement in two different ways, by reducing the fluctuation levels, and by taking energy away from the longer wavelength part of the spectrum.

However, the predicted spectrum shift may not be sufficiently large (as indicated in Fig. 6) to compensate the possible enhancement of turbulence due to the increased density gradient at plasma edge during the $\mathrm{H}$-phase. In fact, our physical model reveals that the source term, depicted by $L_{0}$, is determined not only by the density gradient, but also by the radial derivative of the shear, the so-called $E^{\prime \prime}$ effect. A positive $E^{\prime \prime}$ may reduce the unfavorable effect of the sharp (negative) density variation. This tendency, which does not exhibit a power law, is qualitatively in agreement with linear analyses using $E^{\prime \prime} .^{9}$

Our results also indicate that a larger classical dissipation would reduces the turbulence transport as well as the turbulence intensity. This may provide an explanation for the so-called $Z$-mode, for which the abundant impurity content (enhancing classical transport coefficients) tends to suppress the turbulence intensity and the associated transports. 


\section{Acknowledgments}

This work was supported by the U.S. Department of Energy contract \#De-FG05-80ET 53088. 


\section{Appendix A - The Calculation of the Diffusion Tensor}

We need to evaluate the Cartesian components

$$
D_{i j}^{\mu \nu} \equiv \int_{-\infty}^{t} d t^{\prime}\left\langle\left(\mathbf{b} \times \nabla_{i}\right)^{\mu} \phi\left(\mathbf{r}_{i}(t), t\right)\left(\mathbf{b} \times \nabla_{j}\right)^{\nu} \phi\left(\mathbf{r}_{j}\left(t^{\prime}\right), t^{\prime}\right)\right\rangle
$$

of the diffusion tensor $\mathbf{D}_{i j}$ derived in Sec. III. For convenience we first consider the calculation of an auxiliary quantity

$$
P_{i j}^{\mu \nu} \equiv \int_{-\infty}^{t} d t^{\prime}\left\langle\nabla_{i}^{\mu} \phi\left(\mathbf{r}_{i}(t), t\right) \nabla_{j}^{\nu} \phi\left(\mathbf{r}_{j}\left(t^{\prime}\right), t^{\prime}\right)\right\rangle .
$$

Making use of the Fourier representation

$$
\phi\left(\mathbf{r}_{i}(t), t\right)=\int d \omega_{1} d \mathbf{k}_{1} \exp \left[-i \omega_{1} t+i \mathbf{k}_{1} \cdot \mathbf{r}_{i}(t)\right] \phi\left(\omega_{1}, \mathbf{k}_{1}\right)
$$

one obtains

$$
\begin{aligned}
P_{i j}^{\mu \nu}= & \int_{0}^{\infty} d \tau d \omega_{1} d \omega_{2} d \mathbf{k}_{1} d \mathbf{k}_{2} k_{1}^{\mu} k_{2}^{\nu} H\left(\omega_{1}, \mathbf{k}_{1} ; \omega_{2}, \mathbf{k}_{2} ; t, t-\tau\right) \\
& \exp \left[-i \omega_{1} t-i \omega_{2}(t-\tau)+i \mathbf{k}_{1} \cdot \mathbf{r}_{i}+i \mathbf{k}_{2} \cdot \mathbf{r}_{j}\right]
\end{aligned}
$$

where

$$
H\left(\omega_{1}, \mathbf{k}_{1} ; \omega_{2}, \mathbf{k}_{2} ; t, t-\tau\right) \equiv\left\langle\phi\left(\omega_{1}, \mathbf{k}_{1}\right) \phi\left(\omega_{2}, \mathbf{k}_{2}\right) \exp \left[-i \mathbf{k}_{2} \cdot \int_{t-\tau}^{t} d s \mathbf{b} \times \nabla_{j} \Phi\left(\mathbf{r}_{j}(s), s\right)\right]\right\rangle .
$$

Using the recipe given by $\mathrm{Eq}$. (8) to evaluate the ensemble averages, we have

$$
\begin{aligned}
& H\left(\omega_{1}, \mathbf{k}_{1} ; \omega_{2}, \mathbf{k}_{2} ; t, t-\tau\right)=\int d \omega^{\prime} d \mathbf{k}^{\prime}\left\langle\phi\left(\omega_{1}, \mathbf{k}_{1}\right) \phi\left(\omega^{\prime}, \mathbf{k}^{\prime}\right)\right\rangle \\
& \left\langle\frac{\delta}{\delta \phi\left(\omega^{\prime}, \mathbf{k}^{\prime}\right)} \phi\left(\omega_{2}, \mathbf{k}_{2}\right) \exp \left[-i \mathbf{k}_{2} \cdot \int_{t-\tau}^{t} d s \mathbf{b} \times \nabla_{j} \Phi\left(\mathbf{r}_{j}(s), s\right)\right]\right\rangle \\
& \quad=\left\langle\phi\left(\omega_{1}, \mathbf{k}_{1}\right) \phi\left(\omega_{2}, \mathbf{k}_{2}\right)\right\rangle\left\langle\exp \left[-i \mathbf{k}_{2} \cdot \int_{t-\tau}^{t} d s \mathbf{b} \times \nabla_{j} \Phi\left(\mathbf{r}_{j}(s), s\right)\right]\right\rangle
\end{aligned}
$$




$$
\begin{aligned}
&+ \int d \omega^{\prime} d \mathbf{k}^{\prime}\left\langle\phi\left(\omega_{1}, \mathbf{k}_{1}\right) \phi\left(\omega^{\prime}, \mathbf{k}^{\prime}\right)\right\rangle \\
& \cdot\left\langle\phi\left(\omega_{2}, \mathbf{k}_{2}\right) \exp \left[-i \mathbf{k}_{2} \cdot \int_{t-\tau}^{t} d s \mathbf{b} \times \nabla_{j} \mid \Phi\left(\mathbf{r}_{j}(s), s\right)\right]\right. \\
&\left.\left(-i \mathbf{k}_{2}\right) \cdot \int_{t-\tau}^{t} d s^{\prime} \mathbf{b} \times \nabla_{j} \exp \left[-i \omega^{\prime} s^{\prime}+i \mathbf{k}^{\prime} \cdot \mathbf{r}_{j}\left(s^{\prime}\right)\right]\right\rangle .
\end{aligned}
$$

For a stationary homogeneous turbulence

$$
\left\langle\phi\left(\omega_{1}, \mathbf{k}_{1}\right), \phi\left(\omega_{2}, \mathbf{k}_{2}\right)\right\rangle=\left\langle\left|\phi\left(\omega_{1}, \mathbf{k}_{1}\right)\right|^{2}\right\rangle \delta\left(\omega_{1}+\omega_{2}\right) \delta\left(\mathbf{k}_{1}+\mathbf{k}_{2}\right)
$$

which converts (A4) into

$$
\begin{gathered}
H\left(\omega_{1}, \mathbf{k}_{1} ; \omega_{2}, \mathbf{k}_{2} ; t, t-\tau\right)=\left\langle\exp \left[-i \mathbf{k}_{2} \cdot \int_{t-\tau}^{t} d s \mathbf{b} \times \nabla_{j} \Phi\left(\mathbf{r}_{j}(s), s\right)\right]\right\rangle \\
\cdot\left(\left\langle\left|\phi\left(\omega_{1}, \mathbf{k}_{1}\right)\right|^{2}\right\rangle \delta\left(\omega_{1}+\omega_{2}\right) \delta\left(\mathbf{k}_{1}+\mathbf{k}_{2}\right)\right. \\
+\int_{t-\tau}^{t} d s_{1}\left\langle\phi\left(\omega_{1}, \mathbf{k}_{1}\right)\left(-i \mathbf{k}_{2} \cdot \mathbf{b} \times \nabla_{j} \phi\left(\mathbf{r}_{j}\left(s_{1}\right), s_{1}\right)\right\rangle\right. \\
\cdot \int_{t-\tau}^{t} d s_{2}\left\langle\phi\left(\omega_{2}, \mathbf{k}_{2}\right)\left(-i \mathbf{k}_{2} \cdot \mathbf{b} \times \nabla_{j} \phi\left(\mathbf{r}_{j}\left(s_{2}\right), s_{2}\right)\right\rangle\right) .
\end{gathered}
$$

The quantity $J_{i} \equiv\left\langle\phi\left(\omega_{i}, \mathbf{k}_{i}\right)\left(-i \mathbf{k}_{2} \cdot \mathbf{b} \times \nabla_{j} \phi\left(\mathbf{r}_{j}\left(s_{i}\right), s_{i}\right)\right\rangle\right.$ contained in Eq. (A6) can be written in the Fourier representation,

$$
\begin{aligned}
J_{i} & =\int d \omega^{\prime} d \mathbf{k}^{\prime} \mathbf{b} \cdot\left(\mathbf{k}^{\prime} \times \mathbf{k}_{2}\right) \exp \left(-i \omega^{\prime} s_{i}+i \mathbf{k}^{\prime} \cdot \mathbf{r}_{j}\right) \\
& \cdot\left\langle\phi\left(\omega_{i}, \mathbf{k}_{i}\right) \phi\left(\omega^{\prime}, \mathbf{k}^{\prime}\right) \exp \left[-i \mathbf{k}^{\prime} \cdot \int_{s_{i}}^{t} d s^{\prime} \mathbf{b} \times \nabla_{j} \Phi\left(\mathbf{r}_{j}\left(s^{\prime}\right), s^{\prime}\right)\right]\right\rangle \\
& =\int d \omega^{\prime} d \mathbf{k}^{\prime} \mathbf{b} \cdot\left(\mathbf{k}^{\prime} \times \mathbf{k}_{2}\right) \exp \left(-i \omega^{\prime} s_{i}+i \mathbf{k} \cdot \mathbf{r}_{j}\right) H\left(\omega_{i}, \mathbf{k}_{i} ; \omega^{\prime}, \mathbf{k}^{\prime} ; t, s_{i}\right),
\end{aligned}
$$

and when substituted into $\mathrm{Eq}_{\mathrm{q}}$ ( $\mathrm{A} 6$ ), yields an equation for $H$

$$
H\left(\omega_{1}, \mathbf{k}_{1} ; \omega_{2}, \mathbf{k}_{2} ; t, t-\tau\right)=\left\langle\exp \left[-i \mathbf{k}_{2} \cdot \int_{t-\tau}^{t} d s \mathbf{b} \times \nabla_{j} \Phi\left(\mathbf{r}_{j}(s), s\right)\right]\right\rangle
$$




$$
\begin{gathered}
\cdot\left(\left\langle\left.\phi\left(\omega_{1}, \mathbf{k}_{1}\right)\right|^{2}\right\rangle \delta\left(\omega_{1}+\omega_{2}\right) \delta\left(\mathbf{k}_{1}+\mathbf{k}_{2}\right)\right. \\
+\int_{t-\tau}^{t} d s_{1} d \omega^{\prime} d \mathbf{k}^{\prime} \mathbf{b} \cdot\left(\mathbf{k}^{\prime} \times \mathbf{k}_{2}\right) \exp \left(-i \omega^{\prime} s_{1}+i \mathbf{k}^{\prime} \cdot \mathbf{r}_{j}\right) H\left(\omega_{1}, \mathbf{k}_{1} ; \omega^{\prime}, \mathbf{k}^{\prime} ; t, s_{1}\right) \\
\left.\cdot \int_{t-\tau}^{t} d s_{2} d \omega^{\prime \prime} d \mathbf{k}^{\prime \prime} \mathbf{b} \cdot\left(\mathbf{k}^{\prime \prime} \times \mathbf{k}_{2}\right) \exp \left(-i \omega^{\prime \prime} s_{2}+i \mathbf{k}^{\prime \prime} \cdot \mathbf{r}_{j}\right) H\left(\omega_{2}, \mathbf{k}_{2} ; \omega^{\prime \prime}, \mathbf{k}^{\prime \prime} ; t, s_{2}\right)\right) .
\end{gathered}
$$

It is obvious that

$$
\begin{gathered}
H\left(\omega_{1}, \mathbf{k}_{1} ; \omega_{2}, \mathbf{k}_{2} ; t, t-\tau\right)=\left\langle\exp \left[-i \mathbf{k}_{2} \cdot \int_{t-\tau}^{t} d s \mathbf{b} \times \nabla_{j} \Phi\left(\mathbf{r}_{j}(s), s\right)\right]\right\rangle \\
\cdot\left\langle\left|\phi\left(\omega_{1}, \mathbf{k}_{1}\right)\right|^{2}\right\rangle \delta\left(\omega_{1}+\omega_{2}\right) \delta\left(\mathbf{k}_{1}+\mathbf{k}_{2}\right)
\end{gathered}
$$

is an exact solution of Eq. (A8), because the second factor of the second term on the r.h.s of Eq. (A8) vanishes as a consequence of the $\mathbf{E} \times \mathbf{B}$ nonlinearity. Substituting Eq. (A9) into Eq. (A3), we obtain

$$
\begin{aligned}
P_{i j}^{\mu \nu} & =-\int_{0}^{\infty} d \tau d \omega d \mathbf{k} k^{\mu} k^{\nu} \exp \left[-i \omega \tau+i \mathbf{k} \cdot\left(\mathbf{r}_{i}-\mathbf{r}_{j}\right)\right] \\
& \cdot\left\langle\exp \left[i \mathbf{k} \cdot \int_{t-\tau}^{t} d s \mathbf{b} \times \nabla_{j} \Phi\left(\mathbf{r}_{j}(s), s\right)\right]\right\rangle\left\langle|\phi(\omega, \mathbf{k})|^{2}\right\rangle .
\end{aligned}
$$

To evaluate $P_{i j}^{\mu \nu}$, we consider

$$
U_{j}(\tau, \mathbf{k}) \equiv\left\langle\exp \left[i \mathbf{k} \cdot \int_{t-\tau}^{t} d s \mathbf{b} \times \nabla_{j} \Phi\left(\mathbf{r}_{j}(s), s\right)\right]\right\rangle,
$$

which obeys the equation

$$
\begin{aligned}
\frac{\partial}{\partial \tau} U_{j}(\tau, \mathbf{k})= & \left\langle\exp \left[-i \mathbf{k} \cdot \int_{0}^{\tau} d s \mathbf{b} \times \nabla_{j} \Phi\left(\mathbf{r}_{j}(s), s\right)\right](-i \mathbf{k}) \cdot \mathbf{b} \times \nabla_{j} \Phi\left(\mathbf{r}_{j}(\tau), \tau\right)\right\rangle \\
& =i \mathbf{k} \cdot \mathbf{b} \times \nabla \phi_{0} U_{i}(\tau, \mathbf{k})+i \mathbf{k} \cdot \int d \mathbf{r}^{\prime} d t^{\prime}\left\langle\mathbf{b} \times \nabla_{j} \phi\left(\mathbf{r}_{j}(\tau), \tau\right) \phi\left(\mathbf{r}^{\prime}, t^{\prime}\right)\right\rangle \\
& \cdot\left\langle\frac{\delta}{\delta \phi\left(\mathbf{r}^{\prime}, t^{\prime}\right)} \exp \left[i \mathbf{k} \cdot \int_{0}^{\tau} d s \mathbf{b} \times \nabla_{j} \Phi\left(\mathbf{r}_{j}(s), s\right)\right]\right\rangle
\end{aligned}
$$




$$
\begin{aligned}
& =i \mathbf{k} \cdot \mathbf{b} \times \nabla \phi_{0} U_{j}(\tau, \mathbf{k})-\mathbf{k} \cdot \int_{0}^{\tau} d t^{\prime}\left\langle\mathbf{b} \times \nabla_{j} \phi\left(\mathbf{r}_{j}(\tau), \tau\right) \mathbf{b} \times \nabla_{j} \phi\left(\mathbf{r}_{j}\left(t^{\prime}\right), t^{\prime}\right)\right\rangle \cdot \mathbf{k} U_{j}(\tau, \mathbf{k}) \\
& =i \mathbf{k} \cdot \mathbf{b} \times \boldsymbol{\nabla} \phi_{0} U_{j}(\tau, \mathbf{k})-\mathbf{k} \cdot \mathbf{D}_{j j} \cdot \mathbf{k} U_{j}(\tau, \mathbf{k}) .
\end{aligned}
$$

For large $\tau, \mathbf{D}_{i j}$ becomes a constant. Equation (A12) is then solved to obtain $U_{j}(\tau, \mathbf{k})$ at large $\tau$

$$
U_{j}(\tau, \mathbf{k})=\exp \left(i \mathbf{k} \cdot \mathbf{b} \times \nabla \phi_{0} U_{j} \tau-\mathbf{k} \cdot \mathbf{D}_{j j} \cdot \mathbf{k} \tau\right) .
$$

Substituting Eq. (A13) into Eq. (A10), we obtain

$$
P_{i j}^{\mu \nu}=\int d \omega d \mathbf{k} k^{\mu} k^{\nu} \exp \left[i \mathbf{k} \cdot\left(\mathbf{r}_{i}-\mathbf{r}_{j}\right)\right]\left\langle|\phi(\omega, \mathbf{k})|^{2}\right\rangle G_{j}(\omega, \mathbf{k})
$$

where

$$
\begin{aligned}
G_{j}(\omega, \mathbf{k}) & \equiv-\int_{0}^{\infty} d \tau \exp \left(-i \omega \tau+i \mathbf{k} \cdot \mathbf{b} \times \nabla \phi_{0} \tau-\mathbf{k} \cdot \mathbf{D}_{j j} \cdot \mathbf{k} \tau\right) \\
& =\frac{i}{\omega-\mathbf{k} \cdot \mathbf{b} \times \nabla \phi_{0}-i \mathbf{k} \cdot \mathbf{D}_{j j} \cdot \mathbf{k}}
\end{aligned}
$$

which is known as the averaged one-particle Green's function. Defining

$$
\Pi(\mathbf{k}) \equiv \operatorname{Re} \int d \omega G_{j}(\omega, \mathbf{k})\left\langle|\varphi(\omega, \mathbf{k})|^{2}\right\rangle
$$

and remembering that $\mathbf{D}_{j j}^{\mu \nu}$ is obtained from $P_{i j}^{\mu \nu}$ by replacing $k^{\mu} k^{\nu}$ by $(\mathbf{b} \times \mathbf{k})^{\mu}(\mathbf{b} \times \mathbf{k})^{\nu}$, we derive the final expression,

$$
D_{i j}^{\mu \nu}=\int d \mathbf{k}(\mathbf{b} \times \mathbf{k})^{\mu}(\mathbf{b} \times \mathbf{k})^{\nu} \Pi(\mathbf{k}) \exp \left[i \mathbf{k} \cdot\left(\mathbf{r}_{i}-\mathbf{r}_{j}\right)\right] .
$$

Notice that both $D_{i j}^{\mu \nu}$ and $P_{i j}^{\mu \nu}$ are real, and $\Pi(-\mathbf{k})=\Pi(\mathbf{k})$. For a broadband frequency spectrum $\Pi(k)$ simply reduces to the $\phi$ spectrum, which becomes independent of the diffusion coefficient $\mathbf{D}_{j j}$. From Eq. (A17), we readily see that the diffusion tensor is divergentless, i.e.,

$$
\nabla_{i} \cdot \mathbf{D}_{i j}=\nabla_{j} \cdot \mathbf{D}_{i j}=0
$$




\section{Appendix B - Diffusion Tensor for 'Isotropic' $\Pi(k)$ :}

When $\Pi(k)$ [defined by $\mathrm{Eq} .(\mathrm{A} 16)]$ is taken to be 'isotropic' in the sense discussed in Sec. III [after Eq. (24)], the Cartesian components of the diffusion tensor [Eq. (A17)] become (after angle integration)

$$
\begin{aligned}
& D_{-}^{x x}=2 D\left[1-S_{0}(r)-S_{2}(r) \cos 2 \varphi\right] \\
& D_{-}^{y y}=2 D\left[1-S_{0}(r)+S_{2}(r) \cos 2 \varphi\right] \\
& D_{-}^{x y}=-2 D S_{2}(r) \sin 2 \varphi
\end{aligned}
$$

and

$$
D_{12}^{x x}+D_{21}^{x x}=2 \pi D\left[S_{0}(r)+S_{2}(r) \cos 2 \varphi\right]
$$

where $x \equiv r \cos \varphi, y \equiv r \sin \varphi, J_{0}\left(J_{2}\right)$ is the zeroth (second) order Bessel function, and

$$
D=\pi \int_{0}^{\infty} k^{3} \Pi(k) d k
$$

is a scalar measure of the turbulent diffusion. Out of these we need to compute the operator $\mathcal{S} \equiv \boldsymbol{\nabla} \cdot \mathbf{D}_{-} \cdot \boldsymbol{\nabla}$ appear in Eq. (24). Since $\boldsymbol{\nabla} \cdot \mathbf{D}=0$,

$$
\begin{aligned}
\mathcal{S}= & D_{-}^{x x} \frac{\partial^{2}}{\partial x^{2}}+D_{-}^{y y} \frac{\partial^{2}}{\partial y^{2}}+2 D_{-}^{x y} \frac{\partial}{\partial x} \frac{\partial}{\partial y} \\
= & 2 \pi D\left[\left[\left(1-S_{0}(r)\right]\left(\frac{\partial^{2}}{\partial x^{2}}+\frac{\partial^{2}}{\partial y^{2}}\right)+\cos 2 \varphi S_{2}(r)\left(\frac{\partial^{2}}{\partial x^{2}}-\frac{\partial}{\partial y^{2}}\right)\right.\right. \\
& \left.-2 \sin 2 \varphi S_{2}(r) \frac{\partial}{\partial x} \frac{\partial}{\partial y^{2}}\right] .
\end{aligned}
$$

Making use of the identities

$$
\frac{\partial^{2}}{\partial x^{2}}+\frac{\partial^{2}}{\partial y^{2}}=\frac{1}{r} \frac{\partial}{\partial r} r \frac{\partial}{\partial r}+\frac{1}{r^{2}} \frac{\partial^{2}}{\partial \varphi^{2}},
$$




$$
\begin{aligned}
& x \frac{\partial}{\partial x}+y \frac{\partial}{\partial y}=r \frac{\partial}{\partial r} \\
& \left(x \frac{\partial}{\partial y}-y \frac{\partial}{\partial x}\right)^{2}=\frac{\partial^{2}}{\partial \varphi^{2}}
\end{aligned}
$$

and some Bessel function identities, we obtain

$$
\nabla \cdot \mathbf{D} \cdot \boldsymbol{\nabla}=\mathcal{S}=2 D\left[\frac{1}{r} \frac{\partial}{\partial r}\left(1-S_{0}(r)-S_{2}(r)\right) r \frac{\partial}{\partial r}+\left(1-S_{0}(r)+S_{2}(r)\right) \frac{1}{r^{2}} \frac{\partial^{2}}{\partial \varphi^{2}}\right] .
$$

Substituting (B4) and (B8) into Eq. (24) and dividing by 2D, one obtains Eq. (25) of Sec. III.

In order to write down the explicit values of $\mathcal{S}$ for the model

$$
\Pi(k)=\Pi_{0} e^{-\lambda k^{2}}
$$

we need to calculate

$$
\begin{aligned}
& \Pi_{0} \int_{0}^{\infty} d k k^{3} e^{-\lambda k^{2}}=\frac{\Pi_{0}}{2 \lambda^{2}} \\
& \Pi_{0} \int_{0}^{\infty} d k k^{3} J_{0}(k r) e^{-\lambda k^{2}}=\frac{\Pi_{0}}{2 \lambda^{2}}\left[1-\frac{r^{2}}{4 \lambda}\right] e^{-r^{2} / 4 \lambda} \\
& \Pi_{0} \int_{0}^{\infty} d k k^{3} J_{2}(k r) e^{-\lambda k^{2}}=\Pi_{0} \frac{r^{2}}{(2 \lambda)^{3}} e^{-r^{2} / 4 \lambda}
\end{aligned}
$$

yielding $\left[\rho^{2}=r^{2} / 4 \lambda, \rho=r / 2 \lambda^{1 / 2}\right]$

$$
\begin{gathered}
S_{0}(\rho)=\left(1-\rho^{2}\right) e^{-\rho^{2}}, \\
S_{2}(\rho)=\rho^{2} e^{-\rho^{2}},
\end{gathered}
$$

which are used to obtain Eq. (65). 


\section{Appendix C}

In this appendix, we delineate how to approximate the integral in Eq. (31) to obtain Eq. (33). The demonstration is constructive. Let $\eta^{2}+1-\left(2 / r^{\prime}\right) S_{1}\left(r^{\prime}\right) \equiv D\left(r^{\prime}\right)$, then

$$
A(r)-\frac{1}{L_{0}^{2}} \int_{\infty}^{r} \frac{S_{1}\left(r^{\prime}\right)}{D\left(r^{\prime}\right)} d r^{\prime}
$$

Let us also consider the integral

$$
\hat{A} \equiv-\frac{1}{L_{0}^{2}} \int_{\infty}^{r} \frac{\left(\partial / \partial r^{\prime}\right) D\left(r^{\prime}\right)}{D\left(r^{\prime}\right)} d r^{\prime}=-\left.\frac{1}{L_{0}^{2}} \ln D\left(r^{\prime}\right)\right|_{\infty} ^{r}=-\frac{1}{L_{0}^{2}} \ln \left[\frac{D(r)}{1+\eta^{2}}\right] .
$$

Now we wish to determine the factor $\delta$ such that

$$
\Lambda \equiv A-\delta \hat{A} \ll \delta \hat{A}
$$

so that we could approximate $A \sim \delta \widehat{A}$. Now

$$
\Lambda=-\frac{1}{L_{0}^{2}} \int_{\infty}^{r} d r^{\prime} \frac{\left[S_{1}\left(r^{\prime}\right)-\delta\left(\partial / \partial r^{\prime}\right) D\left(r^{\prime}\right)\right]}{D\left(r^{\prime}\right)} .
$$

Since $D\left(r^{\prime}\right)$ tends to be small for small values of $r^{\prime}$, we know that $\Lambda$ must get its principal contributions from the region $r^{2} \sim \eta^{2} \ll 1$. It is precisely in this region that we can make the numerator very small by choosing appropriately. Since

$$
S_{1}(r)-\delta(\partial / \partial r) D(r) \underset{r \rightarrow 0}{\longrightarrow} \frac{r}{2}\left[1-\frac{\left\langle k_{\perp}^{2}\right\rangle}{8} r^{2}+\mathcal{O}\left(r^{4}\right)\right]-\delta\left[-\frac{\left\langle k_{\perp}^{2}\right\rangle}{4} r+\cdots\right],
$$

the choice $\delta=2 /\left\langle k_{\perp}^{2}\right\rangle$ will eliminate the leading order (proportional to $r$ ) term in the numerator, and make $\Lambda \sim \eta^{2}$. However

$$
\delta \widehat{A}=-\frac{2}{L_{0}^{2}\left\langle k_{\perp}^{2}\right\rangle} \ln \left[1-\frac{(2 / r) S_{1}(r)}{1+\eta^{2}}\right]
$$

goes as $\left|\ell n \eta^{2}\right| \gg \eta^{2}\left(\eta^{2} \ll 1\right)$ in the regions of interest. Therefore $\Lambda \ll \delta \hat{A}$, allows us to approximate

$$
A \simeq-\frac{2}{L_{0}^{2}\left\langle k_{\perp}^{2}\right\rangle} \ln \left[1-\frac{(2 / r) S_{1}(r)}{1+\eta^{2}}\right]
$$

Notice that we could have rewritten 


$$
A=-\frac{2}{L_{0}^{2}\left\langle k_{\perp}^{2}\right\rangle}\left[\ln \left[1+\eta^{2}-\frac{2}{r} S_{1}(r)\right]-\ln \left(1+\eta^{2}\right)\right]
$$

where the second term is of $\mathcal{O}\left(\eta^{2}\right)$. The form $(C 6)$ is however kept because it makes $A$ go to zero at infinity. Neglecting the second term in (C7) would make $A$ approach a small constant value as $r \rightarrow \infty$. This, however, is not physically warranted. 


\section{References}

1 ASDEX TEAM, Nucl. Fusion 29, 1959 (1989).

${ }^{2}$ R.J. Taylor, M.L. Brown, B.D. Fried, H. Grote, J.R. Liberati, G.J. Morales, and P. Pribyl, Phys. Rev. Lett. 63, 2365 (1989).

${ }^{3}$ R.J. Groebner, K.H. Burrel, and R.P. Seraydarian, Phys. Rev. Lett. 64, 3015 (1990).

4 R.D. Stambaugh, S.M. Wolfe, R.D. Hawryluk, J.H. Harris, H. Biglari, S.C. Prager, R.J. Goldston, R.J. Fonck, T. Ohkawa, B.G. Logan, and E. Oktay, Phys. Fluids B 2, 2941 (1990).

${ }^{5}$ K.H. Burrel, T.N. Carlstron, E.J. Doyle, P. Gohil, R.J. Groebner, T. Lehecka, N.C. Luhmann, H. Matsumoto, T.H. Osborne, W.A. Peebles, and R. Philipona, Phys. Fluids B 2, 1405 (1990).

${ }^{6}$ Ch.P. Ritz, H. Lin, T.L. Rhodes, and A. Wootton, Phys. Rev. Lett. 65, 2543 (1990).

7 E.J. Doyle, R.J. Groebner, K.H. Burrel, P. Gohil, T. Lehecka, N.C. Luhmann, H. Matsumoto, T.H. Osborne, W.A. Peebles, and R. Philipona, Phys. Fluids B 3, 2300 (1991).

8 R. Philipona, E.J. Doyle, N.C. Luhmann, W.A. Peebles, C.L. Rettig, R.J. Groebner, K.H. Burrel, P. Gohil, H. Matsumoto, and R. Stambaugh, in Proceedings of the 18th European Conference on Controlled Fusion and Plasma Physics 1991, edited by P. Bachmann and D.C. Robinson (EPS Petti-Lancy, Switherland, 1991), Vol. 15C, Part I, pp. I-281 and A71.

${ }^{9}$ G.M. Staebler and R.R. Dominguez, Nucl. Fusion 31, 1891 (1991). 
10 A.B. Hassam, Comm. Plas. Phys. and Contr. Nucl. Fus. 14, 275 (1992).

11 F. Walbroeck, T.M. Antonsen, Jr., P.N. Guzdar, and A.B. Hassam, Phys. Fluids B 4, $2441(1992)$.

12 K.R. Shaing, G.S. Lee, B.A. Carreras, W.A. Houlberg, and E.C. Crume, Jr., in Proceedings of the Twelfth International Conference on Plasma Physics and Controlled Nuclear Fusion Research, 1988 (International Atomic Energy Agency, Vienna, 1989), Vol. II, p. 13.

13 H. Biglari, P.H. Diamond, and P.W. Terry, Phys. Fluids B 2, 1 (1990).

14 K.R. Shaing, E.C. Crume, Jr., and W.A. Houlberg, Phys. Fluids B 2, 1492 (1990).

15 K.R. Shaing, Comm. Plas. Phys. and Contr. Nucl. Fus. 14, 41 (1991).

16 Y.Z. Zhang and S.M. Mahajan, Phys. Fluids B 4, 1385 (1992).

17 T.H. Dupree, Phys. Fluids 15, 334 (1972).

18 T.H. Dupree, Phys. Fluids 21, 783 (1978).

19 A. Hasegawa and K. Mima, Phys. Fluids 21, 87 (1978).

20 A. Hasegawa, C. Maclennan, and Y. Kodama, Phys. Fluids 22, 2122 (1979).

21 M. Wakatani and A. Hasegawa, Phys. Fluids 27, 611 (1984).

22 Y.Z. Zhang, Acta Physica Sinica 30, 584 (1981).

23 J.F. Drake and A.B. Hassam, Phys. Fluids 24, 1262 (1981).

24 A.B. Hassam and J.F. Drake, Phys. Fluids 26, 133 (1983).

25 Y.Z. Zhang and S.M. Mahajan, Phys. Fluids B 4, 207 (1992). 
${ }^{26}$ T.S. Hahm, P.H. Diamond, P.W. Terry, L. Garcia, and B.A. Carreras, Phys. Fluids 30, $1452(1987)$.

${ }^{27}$ K. Furutsu, J. Res. NBS D-67, 303 (1963).

${ }^{28}$ E.A. Novikov, Sov. Phys.-JETP 20, 1290 (1965).

${ }^{29}$ R. Kraichnan, Phys. Fluids 10, 1417 (1967).

${ }^{30}$ S. Ichimaru, in Basic Principles of Plasma Physics, (W.A. Benjamin, Inc., 1973) and the cited references.

${ }^{31}$ Ch.P. Ritz, private communication.

32 T.H. Dupree, Phys. Fluids 9, 1773 (1966).

${ }^{33}$ S.A. Orzag and R.M. Kraichnan, Phys. Fluids 10, 1720 (1967).

${ }^{34}$ D. Biskamp, Z. Zaturforsch Teil B 23a, 1362 (1968).

${ }^{35}$ L.I. Rudakov and V.N. Tsytovich, Plas. Phys. 13, 213 (1971).

36 T.H. Dupree and D.J. Tetrault, Phys. Fluids 21, 425 (1978).

${ }^{37}$ Y.Z. Zhang and S.M. Mahajan, Phys. Rev. A 32, 1759 (1985).

38 Y.Z. Zhang and S.M. Mahajan, Phys. Fluids 31, 2894 (1987). 


\section{Figure Captions}

1. The suppression factor $S_{p}\left(2 \hat{v}_{y}^{\prime} / 3\right)$ versus $\hat{v}_{y}^{\prime}$ in the asymptotic theory. The solid curve is the result of Eq. (57). The dashed curve is the result from the orbit theory [Eq. (79)]. The dotted-dashed curve is the asymptote of $\left(\hat{v}_{y}^{\prime}\right)^{-2 / 3}$.

(b) The self-consistent turbulence suppression versus $\hat{v}_{y 0}^{\prime}$ in the asymptotic theory. The solid curve is the result of Eq. (58). The dashed curve is the self-consistent result of Eq. (79). The curves $a, b$, and $c$ stand for $\gamma=0 ., 0.5$, and 1. respectively.

2. (a) The angular distribution of the correlation function in the asymptotic theory based on Eqs. (52), (57), and (59). The curves $a, b, c$, and $d$ stand for $\widehat{v}_{y}^{\prime}=1.0,2.5,2.8$, and 3.3 respectively at $\ln r_{0}=-1.0$.

(b) The angular distribution of the correlation function in the asymptotic theory based on Eqqs. (52), (57), and (59). The curves $a, b, c$, and $d$ stand for $\ln r_{0}=-1.0,-1.5,-2.0$, and -2.5 respectively at $\hat{v}_{y}^{\prime}=3.3$.

3. The angular spectrum in the asymptotic theory based on Eq. (63). The amplitude is normalized to 1 . at $\epsilon=0$. The curves $a, b, c$, and $d$ stand for $\widehat{v}_{y}^{\prime}=0 .,-0.5,-1$, and -5.0 respectively.

4. The zeroth harmonic component of the correlation function $A(\rho)$ at $g=-1.0, \eta^{2}=$ 0.01 . The curves $a, b$, and $c$ stand for $\hat{v}_{y}^{\prime}=0 ., 0.2$, and 0.5 respectively.

(b) The $\cos (2 \epsilon)$ component of the correlation function $B_{c}(\rho)$ at $g=-1.0, \eta^{2}=0.01$. The curves $a, b$, and $c$ stand for $\widehat{v}_{y}^{\prime}=0 ., 0.2$, and 0.5 respectively.

(c) The $\sin (2 \epsilon)$ component of the correlation function $B_{s}(\rho)$ at $g=-1.0, \eta^{2}=0.01$. The curves $a, b$, and $c$ stand for $\hat{v}_{y}^{\prime}=0 ., 0.2$, and 0.5 respectively. 
5. The zeroth harmonic component of the spectrum $I^{(0)}(k)$ at $g=-1.0, \eta^{2}=0.01$. The solid curves $a, b$, and $c$ stand for $\widehat{v}_{y}^{\prime}=0 ., 0.2$, and 0.5 respectively. The dotted-dashed curves are the results inferred from the asymptotic theory, where $b^{\prime}$ and $c^{\prime}$ stand for $\hat{v}_{y}^{\prime}=0.2$, and 0.5 respectively.

(b) The $\cos 2 \epsilon$ component of he spectrum $I_{c}(k)$ at $g=-1.0, \eta^{2}=0.01$. The curves $a, b$, and $c$ stand for $\hat{v}_{y}^{\prime}=0 ., 0.2$, and 0.5 respectively.

(c) The $\sin 2 \epsilon$ component of the spectrum $I_{s}(k)$ at $g=-1.0, \eta^{2}=0.01$. The curves $a$, $b$, and $c$ stand for $\widehat{v}_{y}^{\prime}=0 ., 0.2$, and 0.5 respectively.

6. Shift of the mean square of wave number $\bar{k}_{\perp}^{2}$ by shear flow. 


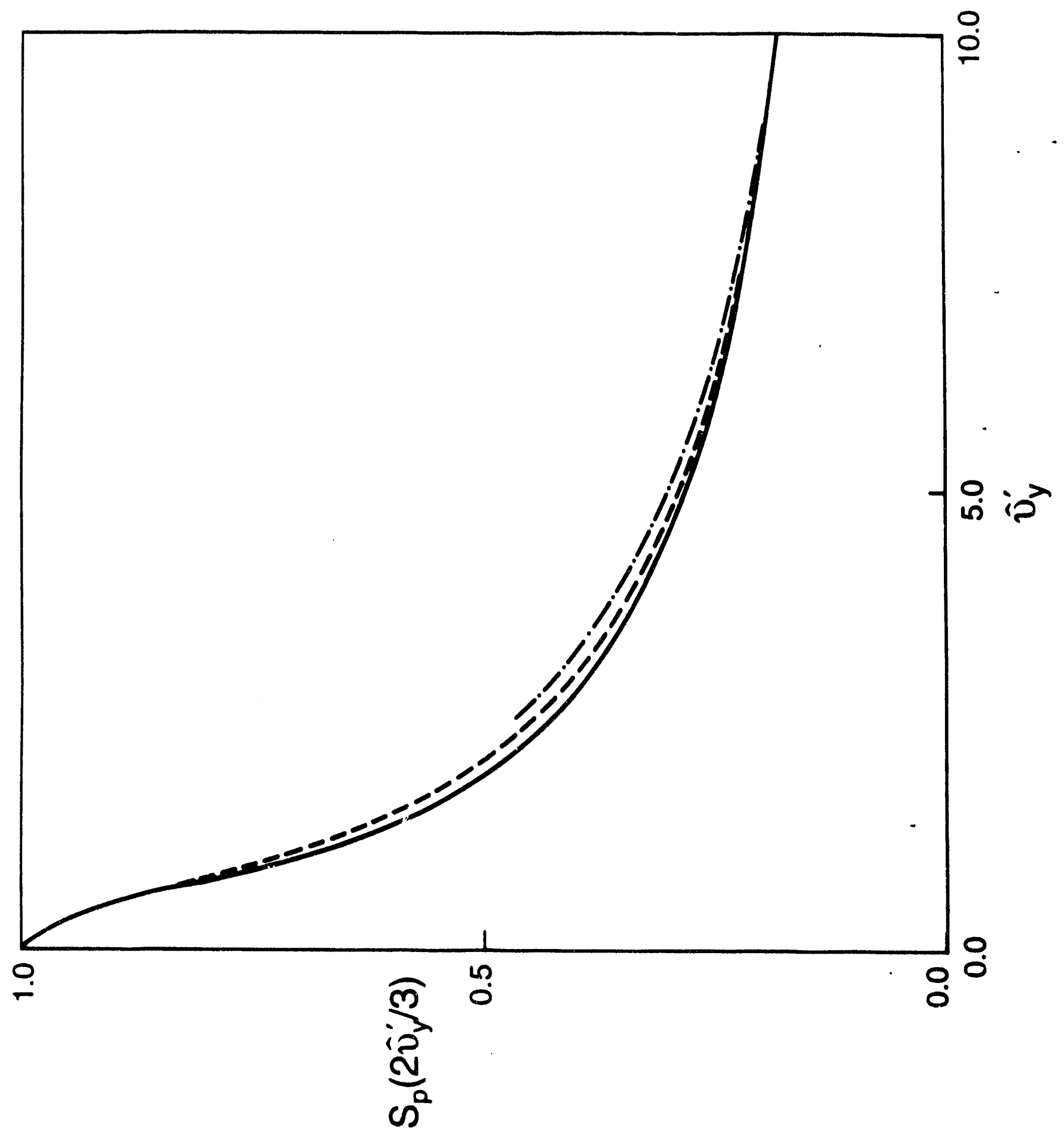

Fig. 1a 


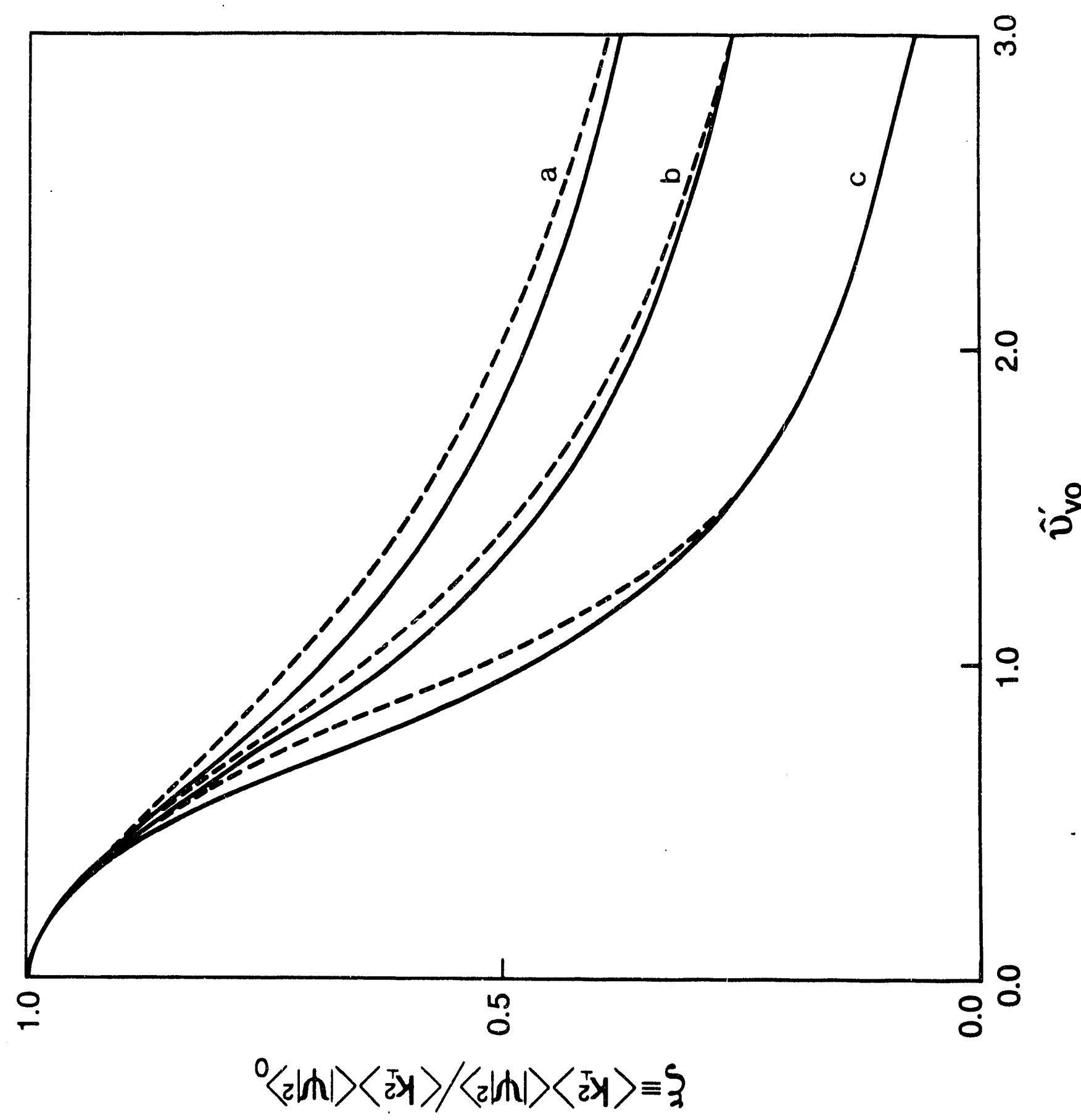

Fig. Ib 


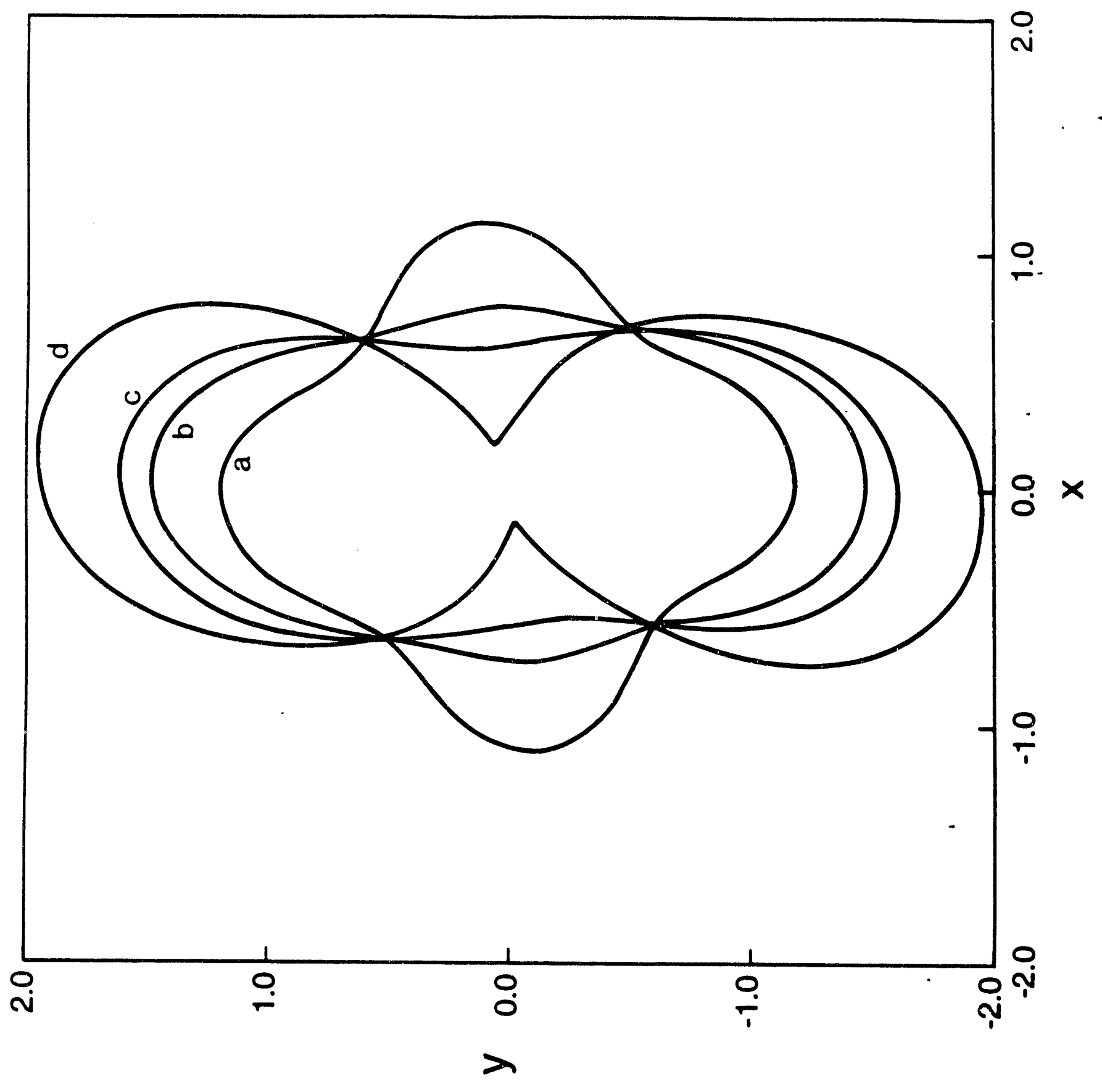

Fig. $2 a$ 


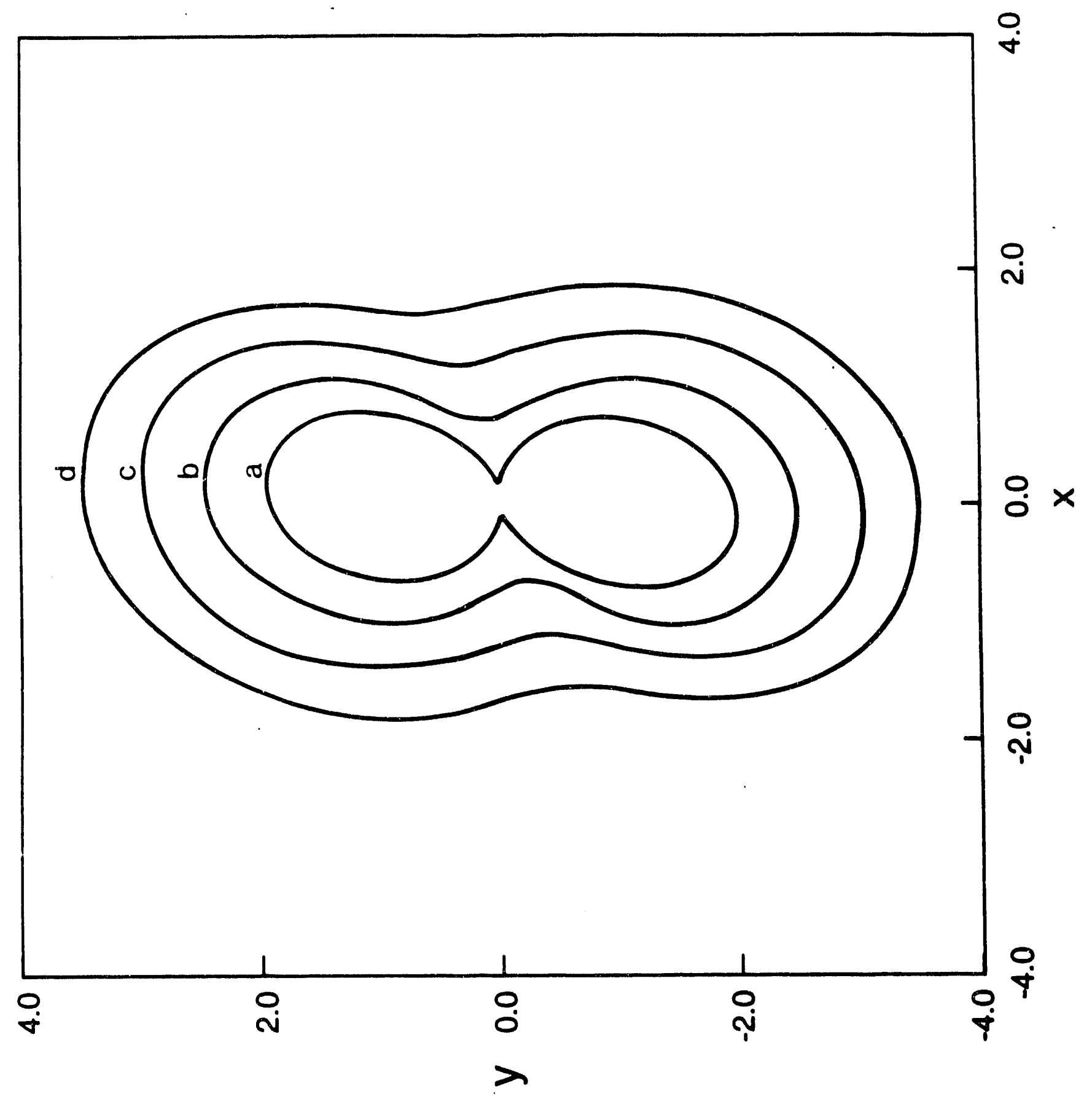

Fig. 2b 


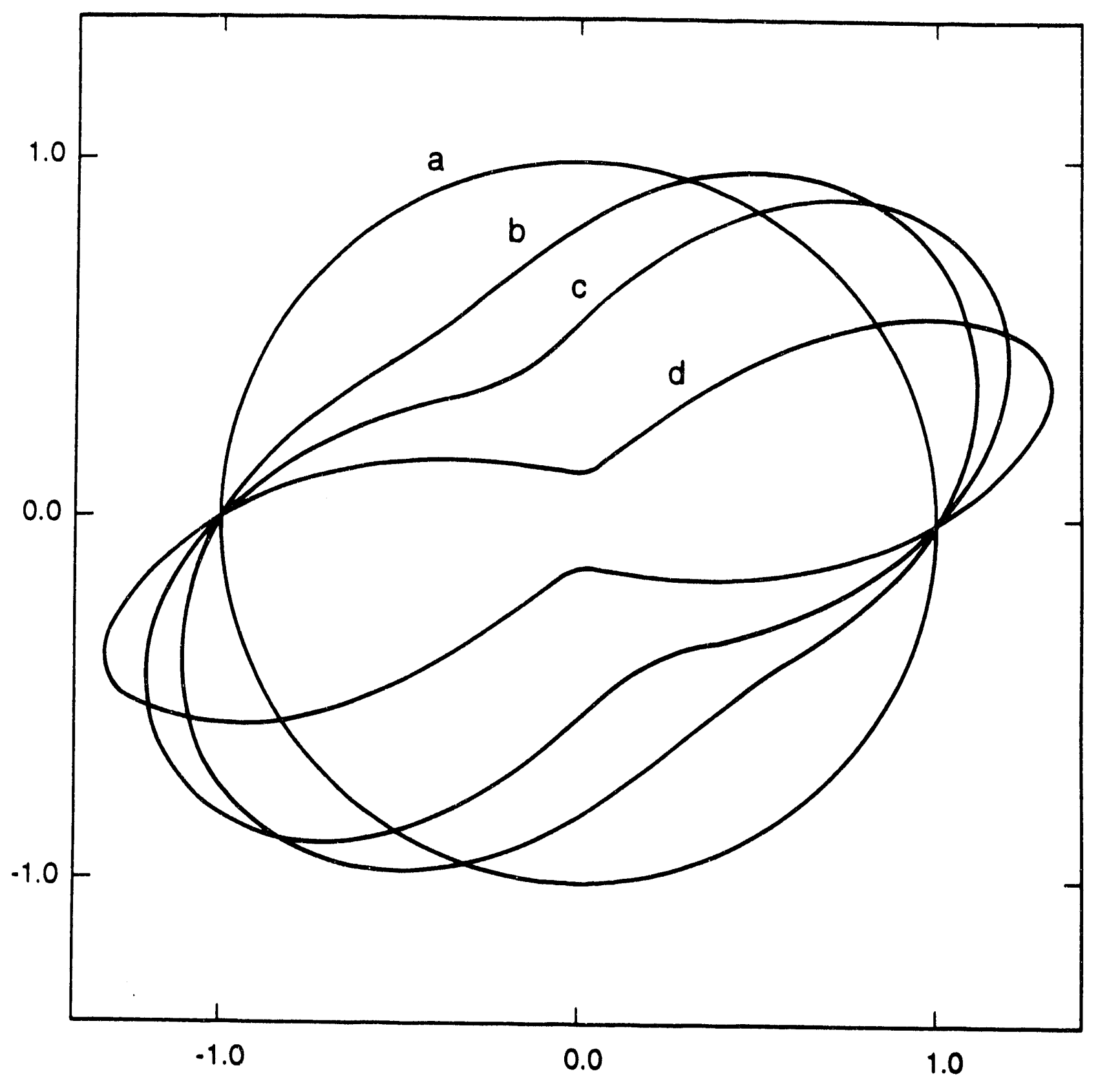

Fig. 3 


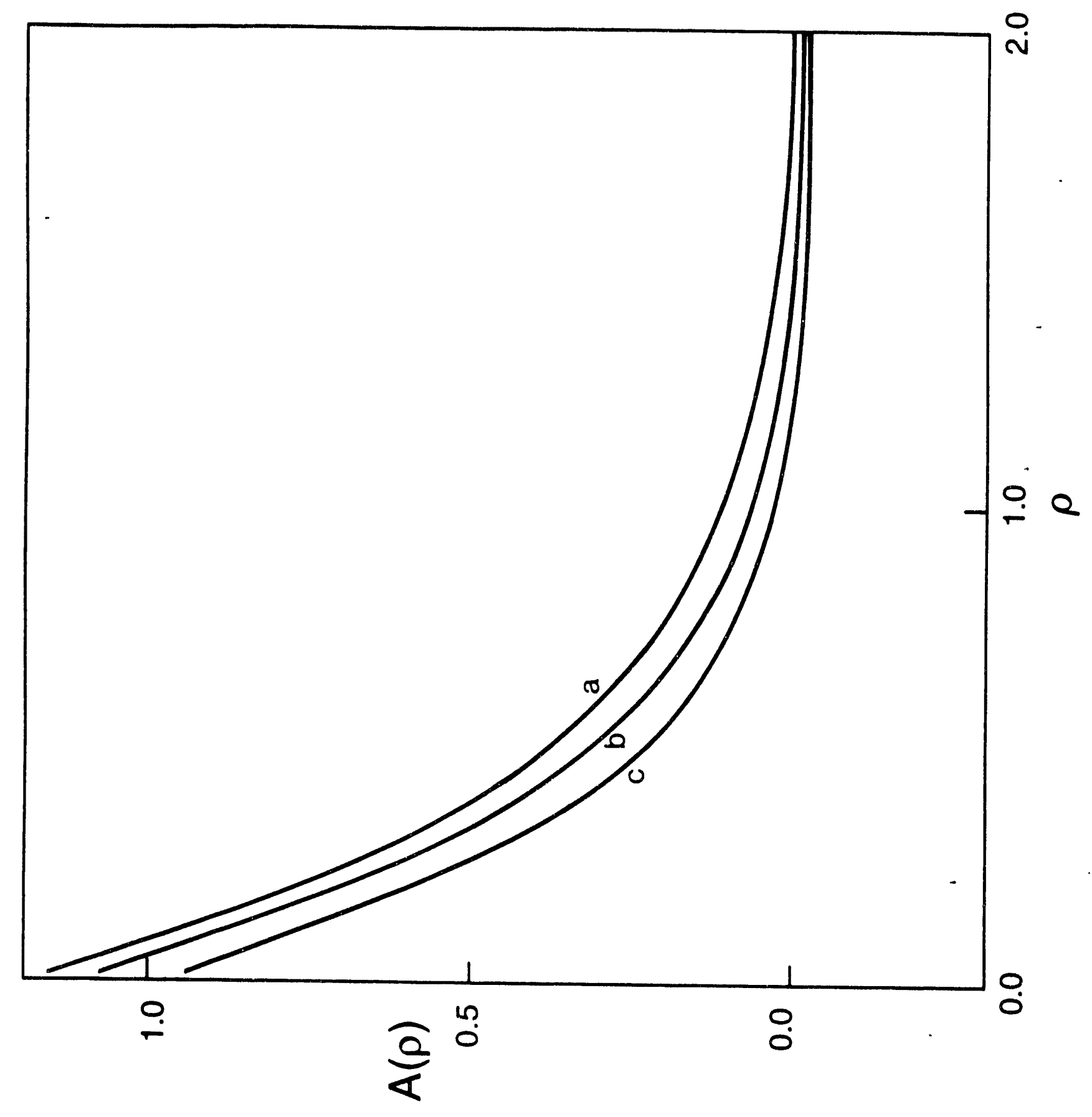

Fig. $4 \mathrm{a}$ 


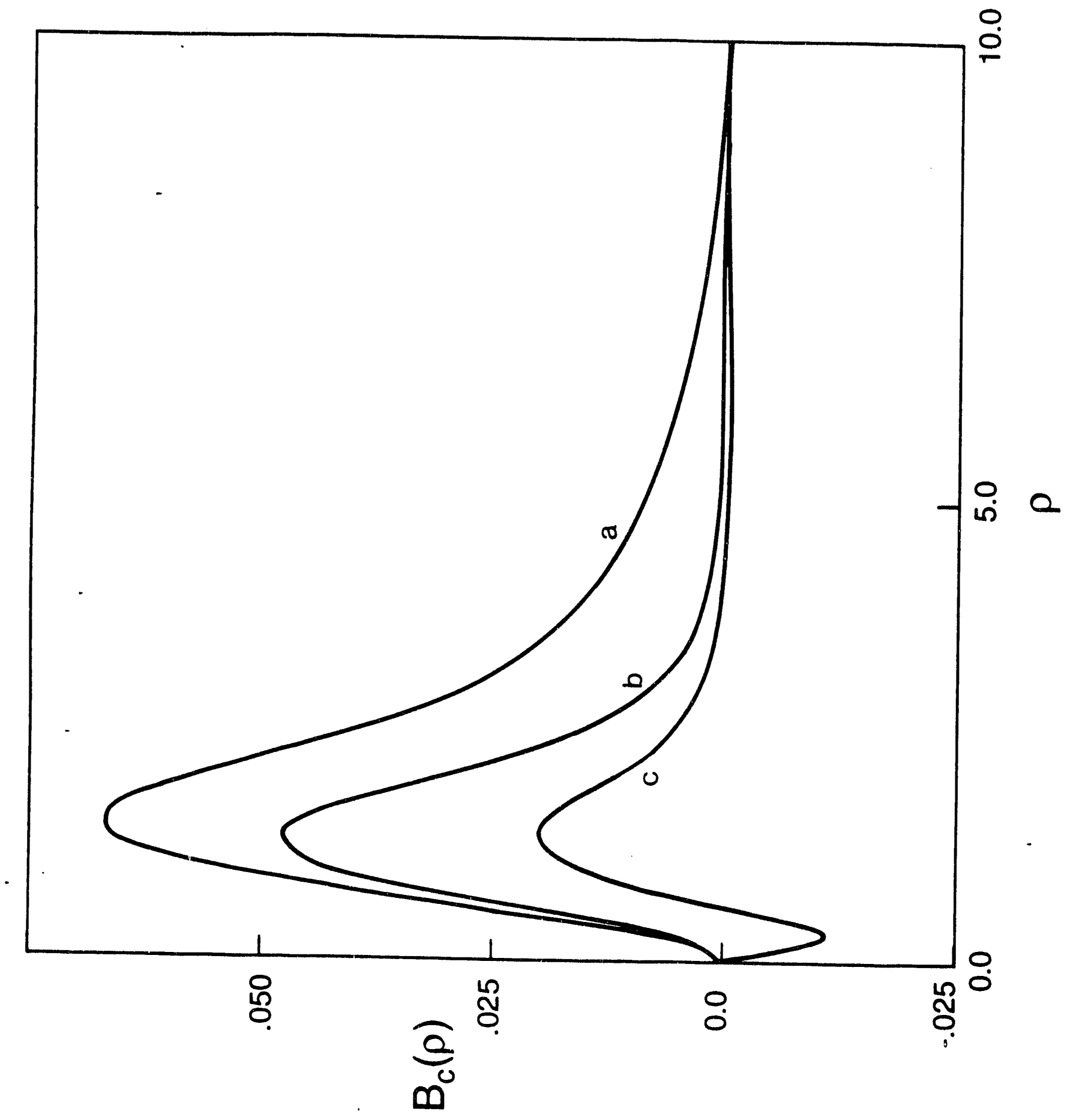

Fig. 4b 


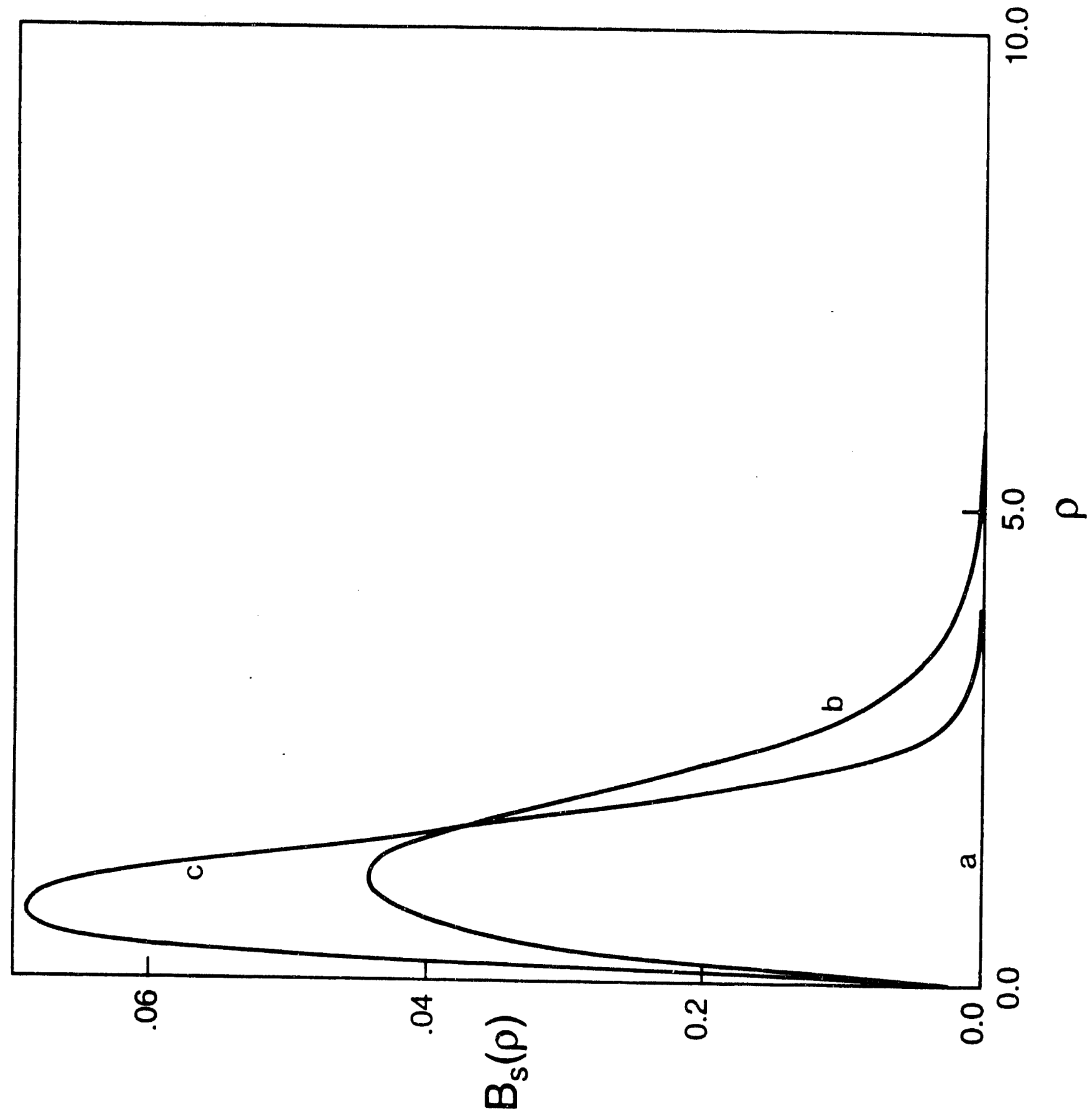

Fig. 4c 


$$
\alpha
$$




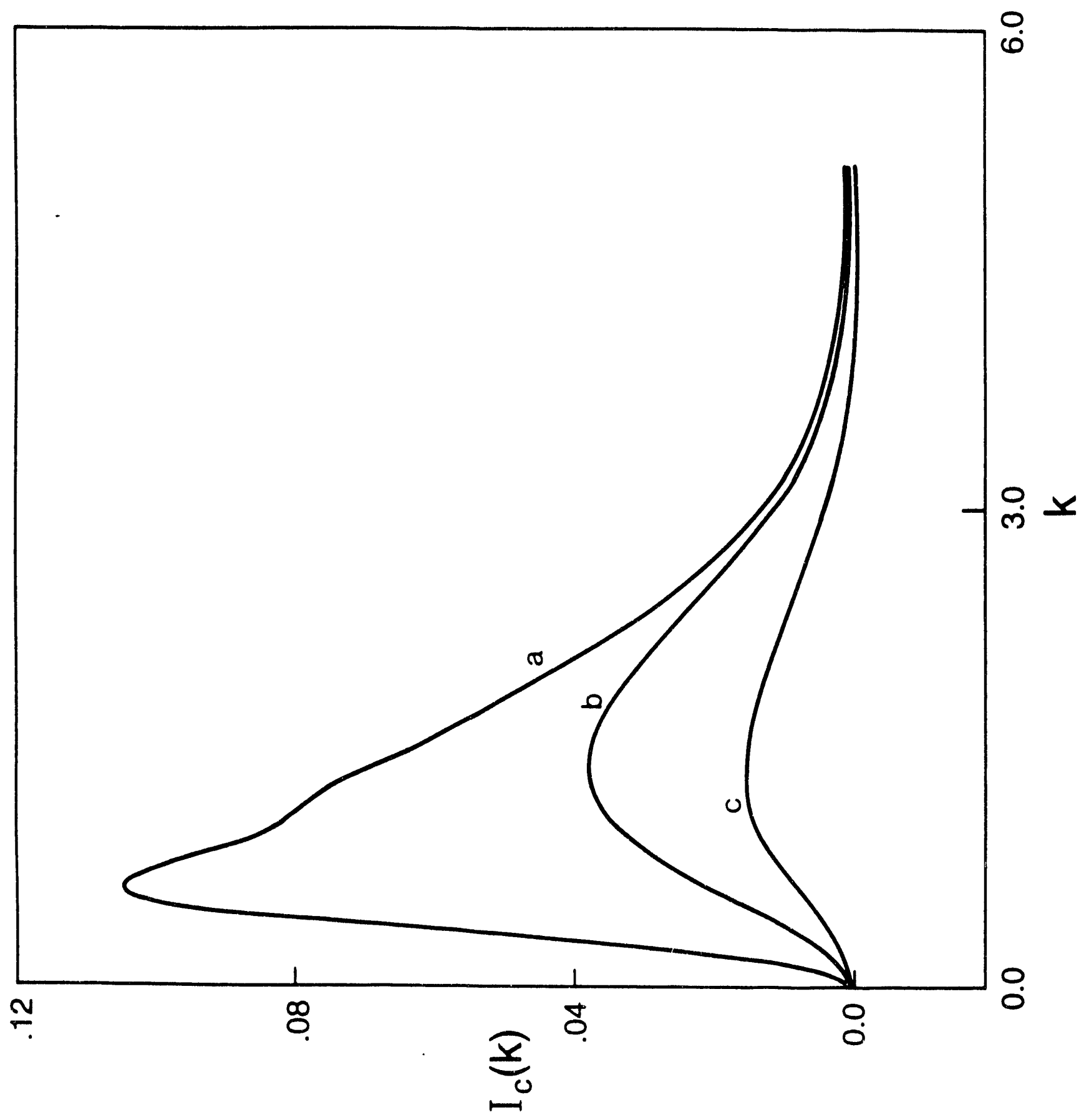

Fig. $5 b$ 


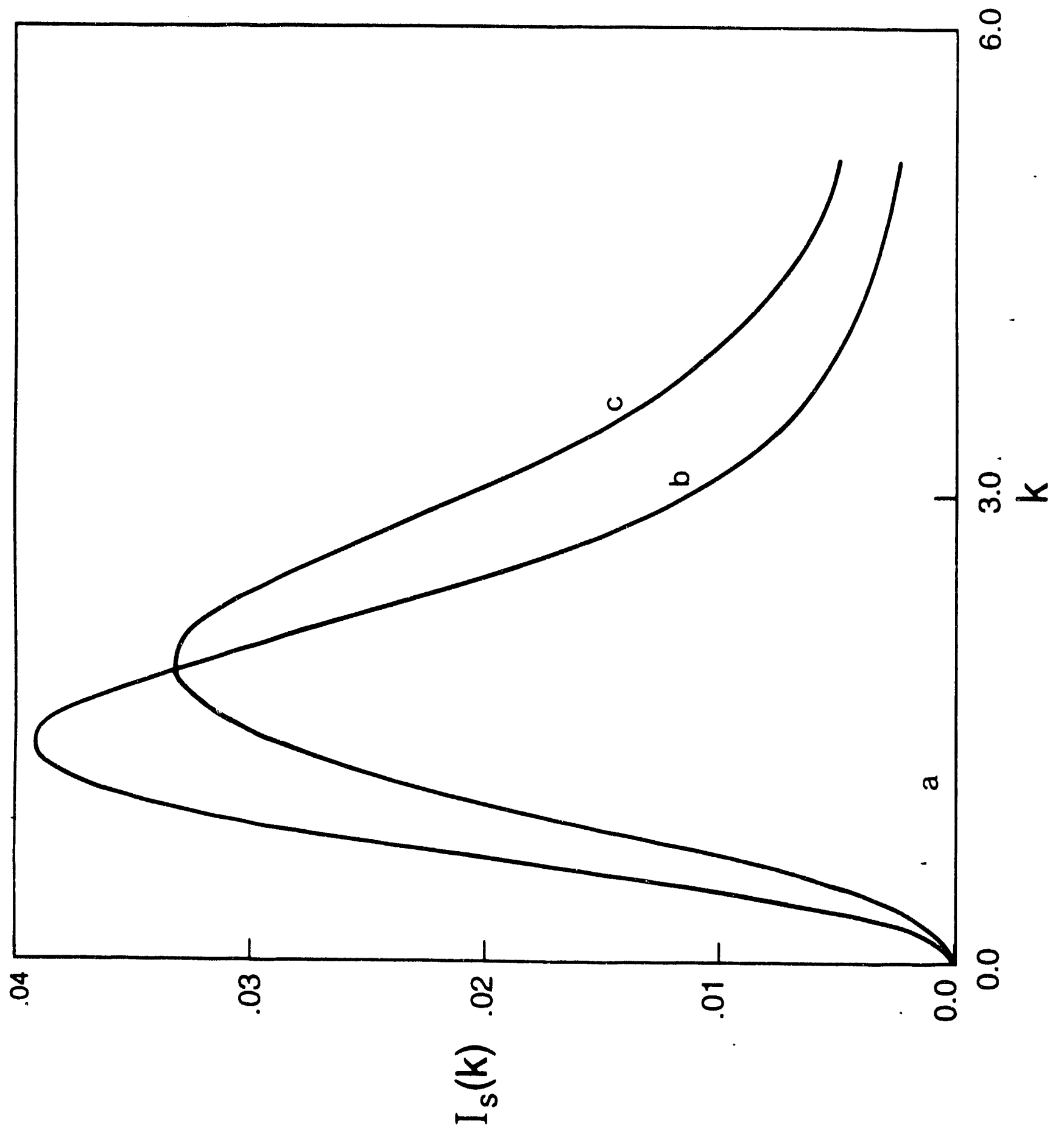

Fig. 5c 


$$
\square
$$



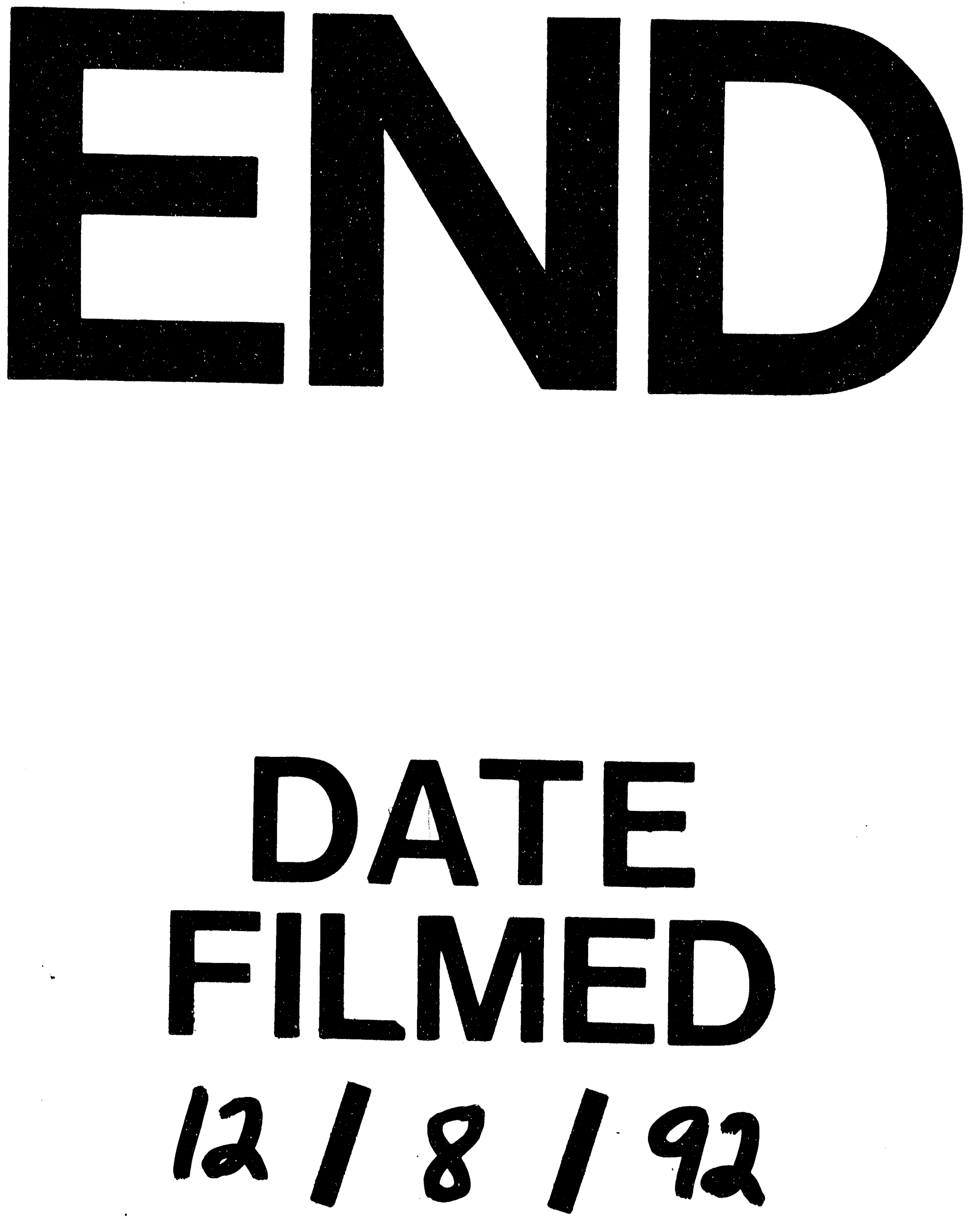
